\title{
Die tirolische Legende vom „Anderl von Rinn“ - Andreaskult und Wallfahrtskirche
}

\author{
Judith Dengler \\ Kerngebiet: Neuzeit \\ eingereicht bei: Univ.-Prof. Dr. Stefan Ehrenpreis \\ eingereicht im: SoSe 2016 \\ Rubrik: SE-Arbeit
}

\section{Abstract \\ The Tyrolean Legend of the "Anderl von Rinn" - the Cult and Pilgrimage Church}

Hippolyt Guarinoni is a key figure in the Tyrolean cult of Anderl. His anti-Jewish attitude was rooted in a deeply Catholic attitude very common in the Baroque era. He created a Tyrolean martyr's cult following the concept of the legend of Simon of Trient. The Anderl legend spread very fast. It was then connected to a child's murder in Judenstein, near Innsbruck, for which Jews were made responsible. To commemorate the violent "ritual death" of Anderl, Guarinoni planned a church at the very spot of his death. The plan was realized after Guarinonis death in the $17^{\text {th }}$ century. Pilgrimages, processions, songs, prayers, plays, etc. made the place famous far across borders. These pilgrimages continued far into the $20^{\text {th }}$ century, thus keeping up a latent antisemitism in the population. The cult was forbidden in 1989 by Bishop Stecher.

\section{Einleitung}

„Wir erkennen nun, daß viele Jahrhunderte der Blindheit unsere Augen bedeckt haben, so daß wir die Schönheit deines auserwählten Volkes nicht mehr sehen und in seinem Gesicht nicht mehr die Züge unseres erstgeborenen Bruders auf unserer Stirn steht. Jahrhundertelang hat Abel darniedergelegen in Blut 
und Tränen, weil wir deine Liebe vergaßen. Vergib uns den Fluch, den wir zu Unrecht aussprechen über den Namen der Juden. Vergib, daß wir dich in ihrem Fleisch zum zweiten Mal kreuzigten."1

Mit diesem Gebet wollte Papst Johannes XXIII. zum Ausdruck bringen, dass das Unrecht, welches dem jüdischen Volk auf vielen Ebenen durch die Jahrhunderte hindurch widerfahren war, nun anerkannt wird. Jüdinnen und Juden hatten für lange Zeit die Funktion als Sündenböcke für alle möglichen und unmöglichen Delikte inne. Gotteslästerung, Hostienschändungen, Brunnenvergiftungen u. v. m. wurde ihnen zu Unrecht angelastet - auch Ritualmorde. Ein ebensolcher Mord soll sich nach Hippolyt Guarinoni im Tiroler Ort Rinn im Jahre 1462 zugetragen haben. Opfer war, laut seiner Niederschrift von 1651, das Kind Andreas Oxner, besser bekannt als das Anderl von Rinn, welches durch sein leidvolles und unverschuldetes Ableben zum Märtyrer erhoben wurde. Die Verehrung des Anderl nahm schon bald nach Bekanntwerden der Schriften Guarinonis die Dimension eines lebendigen Kultes an.

Vor diesem Hintergrund ist es Ziel dieser Arbeit zum Thema "Judentum im Reformationsjahrhundert (1475-1618)", die durch Guarinoni angefachte und geschaffene Legende des Anderl von Rinn als Auslöser eines bis in die heutige Zeit bestehenden, jedoch mittlerweile verbotenen Kultes, darzustellen. Dies soll anhand des Aufzeigens der Legenden von Simon von Trient als „Vorgeschichte“ und der nach deren Schema konstruierten Anderl-Legende geschehen. Im Anschluss wird die Person Hippolyt Guarinonis als Schlüsselfigur für die Entstehung des Andreas-Kultes unter Beschreibung seines Vorgehens beleuchtet. Darüber hinaus soll der zeitliche Verlauf und die Ausbreitung des Ritus und seine Formation unter besonderer Berücksichtigung der Judensteiner Wallfahrtskirche nebst der Darstellung ritueller Praktiken (Wallfahrten, Predigten, Prozessionen, Andachten, Gebete) und Publikationen/Liedern sowie Volksschauspielen, die den Kult ausmachen, angeführt und kontextualisiert werden. Den zeitlichen Rahmen bilden dabei das 17. und 18. Jahrhundert.

Zentrale Fragestellungen sind, ob die Legende des Anderl von Rinn an der Person Guarinonis haftete oder ob er eher als Auslöser eines materiellen und immateriellen Märtyrerkultes gesehen werden muss, der sich über die Zeit hinweg verselbstständigte. Des Weiteren soll geklärt werden, wie sich dieser Kult äußerte bzw. über die Jahrzehnte und Jahrhunderte weitergetragen wurde. Zitate aus Originalquellen aus dem Stiftsarchiv Wilten ergänzen die Literaturrecherche.

Die Literatur- und Quellenlage zum Thema ist breit gefächert, da es auf vielfältige Art und Weise bearbeitet werden kann. Positiv für die Forschung ist, dass der Ort des vermeintlichen Martyriums bekannt ist (Judenstein), Bauten wie die Wallfahrtskirche in Rinn erhalten geblieben sind sowie diverse schriftliche Originalquellen zur Einsicht im Stiftsarchiv Wilten verfügbar sind. Unter den Originalschriften befindet sich etwa die 
unveröffentlichte Handschrift Guarinonis zu Andreas von Rinn. ${ }^{2}$ Was jedoch bei der Literaturrecherche auffiel, ist, dass es zumindest bis Mitte der 1990er-Jahre eine Teilung der Werke in zwei Lager gibt: Ein Teil verherrlicht in seinen Büchern und Schriften das Anderl, trotz Aufklärung und Sistierung der Legende, als Märtyrer. Besonders zahlreich vertreten sind hier Publikationen des suspendierten Kaplans Gottfried Melzer. ${ }^{3}$ Der andere Teil, auf dem der hier vorgebrachte Forschungsstand basiert, weiß um die Fiktion Guarinonis Legende und stellt dies auf verschiedene Themenbereiche bezogen, quellenbasiert und wissenschaftlich fundiert dar.

Aus diesem Grund müssen, um auf den aktuellen Forschungsstand eingehen zu können, einige Persönlichkeiten, die sich um die Erforschung und Aufarbeitung der Anderl-Legende mit dem daraus entstandenen Andreas-Kult bemüht haben, genannt werden. Als maßgeblich für den Beginn einer adäquat-kritischen Beschäftigung mit dem Thema, die erst ab den 1980ern einsetzte, kann der aus München stammende Volkskundler Georg R. Schroubek genannt werden. Schroubek war es, der 1985 mit seinem Aufsatz der "Frage der Historizität des Andreas von Rinn"4 nachging. Es handelt sich dabei zusammen mit anderen Beiträgen von ihm in den Zeitschriften Das Fenster $^{5}$ und der Österreichischen Zeitschrift für Volkskunde ${ }^{6}$ um eine der ausführlichsten und fundiertesten Recherchen zum Anderl-Kult. Überdies bettet Schroubek das eigentliche Thema der Anderl-Legende in ein erweitertes historisches Setting ein, indem er einen Überblick über die in der Neuzeit verbreiteten Ritualmordvorwürfe gegen Jüdinnen und Juden ${ }^{7}$ gibt. Auch Bernhard Fresacher setzt sich in seiner Monographie ${ }^{8}$ und in seiner Dissertation ${ }^{9}$ für eine historisch korrekte Darstellung Andreas Oxners ein und erklärt die Verankerung solcher Ritualmordlegenden im kollektiven Gedächtnis. Daneben finden bei ihm im Besonderen zeitgeschichtliche Diskussionen um den AnderlKult und die Wallfahrtskirche in Judenstein Berücksichtigung. Ein weiterer Durchbruch bezüglich der Erforschung der Ursprünge der Legende ist Heinz Noflatscher ${ }^{10}$ zuzu-

2 Hippolyt Guarinoni, Begrü[n]dte Historj / Der Marter, deß Haillig = / Unschuldigen Khindtß / Andree Von Rinn / So durch die Juden, Im 1462. Jahr / Den 12. tag Julj, dem Christe[n]thumb / Zu Hoon Vnd Spott, Ermördt [...], Innsbruck 1651, Stiftsarchiv Wilten, 38A1.

3 Gottfried Melzer, Das selige Kind Andreas von Rinn ein wahrer Märtyrer der katholischen Kirche, Durach 1989; Gottfried Melzer, Der selige Andreas von Rinn als Helfer und Wundertäter, Durach 1991; Gottfried Melzer, Judenstein im Licht der Wahrheit. Fehlerhaftes, Fehlendes, Bedenkliches und Beleidigendes im Buch "Judenstein - das Ende einer Legende", Bad Hall 1996; Gottfried Melzer (Hrsg.), Wir beten zum seligen Andreas von Rinn. Unschuldiges Kind und Märtyrer. Gebete und Novene zu Ehren des seligen Kindes nebst einem Lebensabriss, Seewen 1994.

4 Georg R. Schroubek, Zur Frage der Historizität des Andreas von Rinn, in: Susanna Buttaroni/Stanislaw Musial (Hrsg.), Ritualmord. Legenden in der europäischen Geschichte, Wien-Köln-Weimar 2003, S. 173-196.

5 Georg R. Schroubek, Zur Verehrungsgeschichte des Andreas von Rinn. Zweiter Teil, in: Das Fenster 20 (1986), Heft 39, S. 3845-3855.

6 Georg R. Schroubek, Andreas von Rinn. Der Kult eines „heiligen Mordopfers“ im historischen Wandel, in: Österreichische Zeitschrift für Volkskunde, 98 (1995), Heft 4, S. 371-396.

7 Georg R. Schroubek, Zur Tradierung und Diffusion einer europäischen Aberglaubensvorstellung, in: Rainer Erb (Hrsg.), Die Legende vom Ritualmord. Zur Geschichte der Blutbeschuldigungen gegen Juden (Dokumente, Texte, Materialien 6), Berlin 1993, S. 17-24.

8 Bernhard Fresacher, Anderl von Rinn. Ritualmord und Neuorientierung in Judenstein 1945-1995 (Mit einem Nachwort von Altbischof Reinhold Stecher), Innsbruck 1998.

9 Bernhard Fresacher, Gedächtnis im Wandel. Eine interdisziplinäre systematisch-theologische Studie über die Verarbeitung von Traditionsbrüchen in der Kirche. Mit dem Fallbeispiel „Judenstein“, theol. Diss. Salzburg 1995.

10 Heinz Noflatscher, Jüdisches Leben in Tirol im 16. und 17. Jahrhundert, in: Thomas Albrich (Hrsg.), Vom Mittelalter bis 1805 (Jüdisches Leben im historischen Tirol 1), Innsbruck-Wien 2013, S. 135-246. 
sprechen. Durch Inanspruchnahme von Visitationsberichten als Quellenmaterial konnte er Wurzeln der Legende nachweisen, bevor Guarinoni erste Überlegungen zum Anderl 1620-1622 niederschrieb. Auch Rainer Erb ${ }^{11}$ und Sigrun Anselm ${ }^{12}$ müssen mit ihren Ausführungen zur Geschichte der Ritualmordbeschuldigungen gegen Jüdinnen und Juden an dieser Stelle genannt werden: Die allgemeineren, zeitlich und geografisch weitläufigen Ausführungen zeigen vor allem, dass die Geschichte des Andreas von Rinn keine „alpenländische Kuriosität"13 war, sondern es sich um organisierte Anschuldigungen gegen Jüdinnen und Juden handelt. Der Rinner Fall wird damit in einen größeren Gesamtzusammenhang gestellt. Daneben geben Beiträge in der Kunsttopographie ${ }^{14}$ und im „Dehio Tirol"15 kunsthistorisch fundierte Auskunft über Bau- und Ausstattungsgeschichte der Wallfahrtskirche in Judenstein.

All diese verschiedenen historischen Aspekte und Forschungsstränge münden schließlich in ein umfassendes und kritisch hinterfragendes Bild der Rinner Märtyrerlegende. Ebenso werden Absichten und Folgen der Erzählung klar dargestellt: das Schüren des Judenhasses in Wort, Tat, Bild, Figur und Architektur und die Etablierung eines Tiroler Kults nach dem Vorbild von Simon von Trient.

\section{Vorgeschichte des Andreas-Kultes}

„Die sogenannten Kindermordlegenden waren ein Teil jener Kampagnen, die durch Jahrhunderte von christlicher Seite aus gegen die Juden geführt wurden, und die schon in früheren Jahrhunderten unsägliches Leid über dieses Volk gebracht haben. In diesem Punkt sehen wir [das Christentum] vor dem Gericht der Weltgeschichte nicht gut aus."16

\subsection{Stellung der Juden}

Die Legendenbildung um Andreas von Rinn ist weit vielschichtiger, als auf den ersten Blick meist angenommen wird. Um die Entstehung des judenfeindlichen AnderlKultes vollends verstehen zu können, ist es daher notwendig, die ideengeschichtliche Stellung der Jüdinnen und Juden in der Zeit des Reformationsjahrhunderts kurz zu skizzieren.

11 Rainer Erb, Drittes Bild: Der „Ritualmord“, in: Julius H. Schoeps/Joachim Schlör (Hrsg.), Antisemitismus. Vorurteile und Mythen, München 1995, S. 74-79.

12 Sigrun Anselm, Angst und Angstprojektion in der Phantasie vom jüdischen Ritualmord, in: Rainer Erb (Hrsg.), Die Legende vom Ritualmord. Zur Geschichte der Blutbeschuldigung gegen Juden (Dokumente, Texte, Materialien 6), S. 253-265.

13 Fresacher, Anderl von Rinn, S. 7.

14 Petra Kapferer-Prinoth/Martin Kapferer, Rinn: Wallfahrtskirche Mariä Heimsuchung in Judenstein, in: Reinhard Rampold (Hrsg.), Kunstführer Tirol. Die 400 bedeutendsten Kunstschätze in Nord- und Osttirol, Innsbruck 2014, S. $146-147$.

15 Herta Öttl/Josef Franckenstein u. a., Rinn: Ehem. Wallfahrtskirche, in: Institut für österreichische Kunstforschung des Bundesdenkmalamtes (Hrsg.), Dehio Tirol (Dehio-Handbuch. Die Kunstdenkmäler Österreichs. Topographisches Denkmälerinventar. Tirol), 1980 Wien, S. 655-657.

16 Josef Gelmi, Geschichte der Kirche in Tirol. Nord-, Ost- und Südtirol, Innsbruck 2001, S. 100. 
Bereits im Spätmittelalter und der frühen Neuzeit wurden Jüdinnen und Juden aus ideologisch-religiösen (unchristliche Minderheit, "Gottesmörder", „Hostienschänder") ${ }^{17}$ oder wirtschaftlichen Gründen (Konkurrenz, „Wucherer") ${ }^{18}$ von Katholik_innen angefeindet. Die Folgen waren Außenseitertum, Vertreibung und Schlimmeres. Von der Reformation versprachen sie sich eine Verbesserung ihrer Situation, da Luthers Haltung gegenüber den Jüdinnen und Juden in den ersten Jahren seiner reformatorischen Tätigkeit wohlwollend war. Er propagierte Jüdinnen und Juden freundlich anzunehmen und nicht als "Hunde und Unmenschen"19 zu behandeln, mit dem Hintergedanken, dass sie sich unter diesen Umständen der evangelischen Kirche anschließen würden. Doch die Haltung des Reformators gegenüber den „Sabbatern“20 änderte sich relativ rasch ins Negative, als er feststellen musste, dass eine Glaubensbekehrung der Jüdinnen und Juden nicht eintrat. Dies äußerte sich in judenfeindlichen Schriften Luthers (z. B.: "Von den Juden und ihren Lügen", 1543) ${ }^{21}$, in denen er die Jüdinnen und Juden etwa als Brunnenvergifter und Mörder anprangerte. Eine verstärkte Judenvertreibung und -reglementierung auch in protestantischen Gebieten waren die Folgen.22

Die Ausgrenzung und Anfeindung der jüdischen Bevölkerung fand also in beiden Konfessionen gleichermaßen statt. Das Judentum wurde als auszuschaltende Gefahr für die Christenheit gewertet. ${ }^{23}$ Bemerkenswert ist allerdings, dass der Antijudaismus speziell zur Zeit der Gegenreformation als katholisch-konfessionalisierendes Instrument galt. ${ }^{24}$

\subsection{Ritualmord}

Neben den bereits exemplarisch angeführten gängigen Beschuldigungen gegen Jüdinnen und Juden gab es im Speziellen auch den Vorwurf des Ritualmordes. Erste dahingehende Vorwürfe kamen bereits im 12./13. Jahrhundert ${ }^{25}$ auf. Sie wurden vom katholischen Klerus selbst, der die Jüdinnen und Juden auf theologischer Basis der "Passions- und Osterliturgie"26 als "Christusmörder" verurteilte, initiiert und propagiert.27 Der Tatverdacht lautete dabei, dass Jüdinnen und Juden (Gedanke des Kollektivs!) christliche Opfer entführten oder kauften und grausam zu Tode quälen würden, um die Passion Christi nachzustellen und zu verhöhnen (erste Beschuldigung in nachantiker Zeit: 1144 in Norwich). ${ }^{28}$

17 Isabella Holzmann, Vier Ritualmordlegenden im Vergleich. Anderl von Rinn, Simon von Trient, Ursula Pöck von Lienz, Franz Locherer von Montiggl. mit besonderer Berücksichtigung des Andreas von Rinn, phil. Dipl. Innsbruck 2013, S. 14 und S. 41-55.

18 Holzmann, Vier Ritualmordlegenden im Vergleich, S. 20.

19 Martin H. Jung, Reformation und Konfessionelles Zeitalter (1517-1648), Göttingen-Oakville 2012, S. 232.

20 Jung, Reformation und Konfessionelles Zeitalter, S. 234.

21 Holzmann, Vier Ritualmordlegenden im Vergleich, S. 20.

22 Jung, Reformation und Konfessionelles Zeitalter, S. 231-237.

23 Horst Jungiger, Talmudhetze, in: Wolfgang Benz (Hrsg.), Ereignisse, Dekrete, Kontroversen (Handbuch des Antisemitismus. Judenfeindschaft in Geschichte und Gegenwart 4), Berlin-Boston 2011, S. 406-408, hier S. 406.

24 Noflatscher, Jüdisches Leben in Tirol, S. 218.

25 Gelmi, Geschichte der Kirche in Tirol, S. 99.

26 Schroubek, Zur Tradierung und Diffusion, S. 18.

27 Ebd., S. 17-19.

28 Birgit Tabernig, Die Irrationalität der Ritualmordlegenden. Entwicklungen und Hintergründe jüdischer Ritual- 
Als die Ritualmordlegenden begannen sich im Römisch-Deutschen Reich zu etablieren, kam zu dem Vorwurf des Ritualmordes jener der Blutbeschuldigung hinzu. ${ }^{29}$ Neben der Persiflage der Marter und des Todes von Jesus Christus ging es, so der Glaube, nun auch um die Gewinnung von Christenblut. Die Jüdinnen und Juden, so die Unterstellung, würden bevorzugt reines Kinderblut für medizinische Zwecke und rituelle bzw. magische Praktiken benötigen. Weitere (frühzeitlichere) Ideen sind diesbezüglich auch, dass die Getöteten als „Opferlamm-Ersatz”, das nach jüdischem Brauch zum Pessachfest geschlachtet wurde, dienten. ${ }^{30}$ Trotz aller Irrationalität ${ }^{31}$ und wiederholten Auftretens von Päpsten gegen den Tatbestand des Ritualmordes und des Verbots gegen derartige Anschuldigungen kam es immer wieder dazu. ${ }^{32}$

Als erster Ritualmordfall in Bezug auf Blutbeschuldigungen in Tirol ist jener Ursula Pöcks zu nennen (1442). Das dreijährige Mädchen soll von Jüdinnen und Juden zu Tode gemartert worden sein - die angeblich schuldigen Frauen, Männer und Jugendlichen wurden zwangsgetauft - oder schlimmer - gefoltert und umgebracht. Durch den Verlust von Akten geriet die Legende der kleinen Ursula jedoch bald in Vergessenheit. ${ }^{33}$ Ihre Reaktivierung erfolgte erst wieder durch das Aufdecken eines anderen vermeintlichen Ritualmordes in Norditalien: den Fall des Simon von Trient. ${ }^{34}$

Der zweijährige Simon Unschuldig galt ab dem 23. März 1475 als vermisst. Diverse Suchaktionen entlang der örtlichen Flusskanäle seitens seiner Eltern und anderer Trentiner_innen blieben erfolglos. Am Ostersonntag, einen Tag nach dem traditionellen jüdischen Pessachfest, fand jedoch ausgerechnet eine jüdische Familie das leblose Kind in ihrer Zisterne. Trotz der Befürchtung, als Täter zu gelten, meldete der Vorsteher der jüdischen Gemeinde Trients, Samuel, den Leichenfund sogleich dem Bischof von Trient, Johannes Hinderbach, und Vertretern des Erzherzogs Sigismund von Tirol. Es folgten gegenseitige Beschuldigungen zwischen Jüdinnen und Juden und Christen_innen, wer das Kind mit welchem Motiv ermordet und in die Zisterne gebracht haben könnte. Resultat war die Verhaftung der gesamten jüdischen Gemeinde, die durch Zeugenaussagen von Christen_innen und jüdischen Konvertit_innen schwer belastet wurde. Die Zeug_innen sagten aus, dass sie Schreie aus dem betroffenen jüdischen Haus gehört hätten. Ein Konvertit bestätigte sogar, dass Jüdinnen und Juden Blut für ihre rituellen Handlungen brauchen würden. Diese Aussagen konnten den Bischof von der Schuld der Jüdinnen und Juden überzeugen, woraufhin sie peinlich befragt wurden und in Folge in Haft starben, hingerichtet oder als mildestes Mittel

mordlegenden des Spätmittelalters und der frühen Neuzeit mit besonderer Berücksichtigung der Lienzer Beschuldigung von 1442/43, phil. Dipl. Innsbruck 2000, S. 35-36.

30 Roland T. Poli, Ritualmordlegenden in Tirol. Mit besonderer Berücksichtigung des Falles Franz Thomas Locherr (1744), in: Robert Rebitsch/Elena Taddei (Hrsg.), Politik - Konflikt - Gewalt (Innsbrucker Historische Studien 25), Innsbruck 2007, S. 295-319, hier S. 297-298.

31 Allein schon wegen des Bluttabus im Judentum sind die Vorwürfe nicht zu halten. Dazu: Erb, Drittes Bild, S. 74-79; Hermann L. Strack, Das Blut im Glauben und Aberglauben der Menschheit. Mit besonderer Berücksichtigung der „Volksmedizin“ und des „jüdischen Blutritus", München 1900 
zwangsgetauft wurden. ${ }^{35}$ Diejenigen, die überlebten, wurden vertrieben - und die Synagoge zerstört. ${ }^{36}$ Dies alles geschah, obwohl zu jener Zeit durch ein Dekret von Papst Innozenz IV. (1247) das Führen von Ritualmordprozessen und damit einhergehend von Judenverfolgung verboten war. Nichtsdestotrotz hatte Bischof Hinderbach nicht nur dagegen verstoßen, sondern durch die Art seines Vorgehens einen fairen Prozess verhindert. ${ }^{37}$ Sein einziges Anliegen war es, die Schuld der Jüdinnen und Juden und die Wahrhaftigkeit von Ritualmorden zu demonstrieren. ${ }^{38}$

Des Weiteren begründete Hinderbach, der von der grundsätzlichen Schuld der Jüdinnen und Juden überzeugt war, zwischen Mai 1475 und Juni 1476 einen Kult um das vermeintliche Märtyrerkind Simon. Ihm wurden innerhalb eines knappen Jahres 129 Wunder zugeschrieben. ${ }^{39}$ Der Leichnam Simons wurde schließlich unter reger Anteilnahme des Volkes in der Stadtkirche St. Peter, in einer dort eigens errichteten Kapelle, beigesetzt. ${ }^{40}$ Der Fall Simons von Trient, bei dem die Existenz von jüdischen Ritualmorden „bewiesen“ wurde, erhielt durch Papst Sixtus V. 1588 eine allgemeine Kulterlaubnis (Messe und Brevier) für Trient und gilt seither als Schlüsselereignis in der Geschichte der antijüdischen Ritualmordvorwürfe. ${ }^{41}$ Das Geschehnis aus Trient verbreitete sich durch den neu etablierten Buchdruck ${ }^{42}$ und durch das landesherrliche Einwirken von Sigismund von Tirol und Kaiser Maximilian I.43

In der Causa Simon von Trient, wie auch in allen anderen Fällen von Ritualmordbeschuldigungen, ist klar festzustellen, dass die Verbreitung dieser Vorstellungen von Klerikern und der Bildungsschicht ausging. Die Vorwürfe gegen die Jüdinnen und Juden und die bei der Bevölkerung damit bewusst ausgelösten Ängste ${ }^{44}$ wurden von ihnen instrumentalisiert, um z. B. Wallfahrten zu etablieren und die Ausschaltung ökonomischer Konkurrenz herbeizuführen. Als Konsequenz dieser Strategien fand die Verankerung derartiger antijüdischer Propaganda im kollektiven Gedächtnis statt. ${ }^{45}$ Eine verstärkte „Volkskanonisation"46 bewirkten Legenden, bei denen es vermeintliche Reliquien gab, die von der Bevölkerung verehrt werden konnten. ${ }^{47}$

Diese Faktoren, wie auch die Erzählung der Märtyrerlegende Simons von Trient, flossen in die tirolische Legende des Anderl von Rinn mit ein. Die Auswirkungen der von

35 David L. Dahl, Ritualmordvorwurf in Trient (1475), in: Wolfgang Benz (Hrsg.), Ereignisse, Dekrete, Kontroversen (Handbuch des Antisemitismus. Judenfeindschaft in Geschichte und Gegenwart 4), Berlin-Boston 2011, S. 356357.

36 Gelmi, Geschichte der Kirche in Tirol, S. 100

37 Erst 1478 wurde durch eine Bulle Papst Sixtus'IV. die Führung von Ritualmordprozessen wieder erlaubt, wobei die Dekrete von Innozenz IV. beibehalten wurden. Dazu: Dahl, Ritualmordvorwurf in Trient (1475), S. 357-358.

38 Ebd., S. 356-358.

39 Ebd., S. 357.

40 Gelmi, Geschichte der Kirche in Tirol, S. 100.

41 Ebd., S. 101

42 Das erste Druckwerk, das im neuzeitlichen Tirol erschien, beschäftigte sich mit der Märtyrerlegende des Simon von Trient. Dazu: Poli, Ritualmordlegenden in Tirol, S. 300.

43 Dahl, Ritualmordvorwurf in Trient (1475), S. 358.

44 Anselm, Angst und Angstprojektion, S. 253-265.

45 Poli, Ritualmordlegenden in Tirol, S. 297-301.

46 Schroubek, Zur Tradierung und Diffusion, S. 22.

47 Ebd 
Hippolyt Guarinoni rund hundertfünfzig Jahre nach Vorbild des Trienter Simon-Kultes etablierten Märtyrerlegende reichen dabei bis in die heutige Zeit.

\section{Fundamente des Andreas-Kultes}

„O Gott, aus Verachtung gegen Dich haben ungläubige Juden den unschuldigen Knaben und Märtyrer Andreas der bittersten Art von Leiden unterworfen $[\ldots]]^{\prime \prime 48}$

\subsection{Legende des Anderl von Rinn}

Etwa 15 km von Innsbruck entfernt liegt die Mittelgebirgsgemeinde Rinn. Im Ortsteil Judenstein erinnert eine Wallfahrtskirche daran, was einst dort über dem „Judenstein“ passiert sein soll: Am 12. Juli 1462 machte sich die in Rinn ansässige Tagelöhnerwitwe Maria Oxner zur Feldarbeit nach Ambras auf. Sie bat den Taufpaten ihres knapp dreijährigen Kindes, Hannes Mayr, in ihrer Abwesenheit auf das Kind zu achten, was dieser versprach. Jüdische Kaufleute, die Rinn passierten, boten Hannes Mayr Geld und Spirituosen, wenn er ihnen das Kind überlassen würde. Sie versprachen, es gut zu erziehen und zu einem begüterten Mann zu machen. Hannes Mayr nahm die Goldstücke, die sie für Andreas boten, an und die Kaufleute zogen mit dem Jungen weiter. In einem nahen Wald angekommen zogen sie dem Anderl das Gewand aus, knebelten inn und legten das Kind auf einen großen Stein („Judenstein“). Nachdem sie ihm überall am Körper tiefe Schnittwunden zugefügt hatten, sodass das Kind verblutete, hängten sie den leblosen Leib noch an einen Baum, bevor sie den Ort verließen.

Im Augenblick der Marter ihres Kindes spürte Maria Oxner während der Arbeit am Feld, dass etwas mit ihrem Sohn nicht stimmen konnte. Wie zur Bestätigung ihrer Vorahnung fielen ihr nacheinander drei Blutstropfen auf die Hand, worauf sie sofort zurück nach Rinn eilte, um nach Andreas zu sehen. Dort angekommen stellte sie sogleich Hannes Mayr zur Rede. Dieser wollte ihr den Hut voller Goldstücke zeigen. Die Münzen hatten sich aber in Laub verwandelt. Hannes Mayr verfiel dem Wahnsinn. Maria Oxner suchte nach ihrem Buben und fand ihn über einer Blutlache, an jenem Baum über dem Judenstein, an dem ihn die Juden aufgehängt hatten. Der Leichnam des Anderl von Rinn wurde unter großer Anteilnahme am Rinner Friedhof beigesetzt. An seinem Grab und seinem Marterort ereigneten sich immer wieder Wunder. ${ }^{49}$ Das war der Anlass für den Bau einer Wallfahrtskirche in Judenstein. ${ }^{50}$ Die Gebeine des Kindes, die bereits nach dem Bekanntwerden des Martyriums des Simon von Trient in die alte

\footnotetext{
48 Melzer, Wir beten zum seligen Andreas von Rinn, S. 44.

49 Neben Wundern seien dem Grab eine Lilie, die eine Schrift in ihrer Knospe barg, aber von Unholden gepflückt worden sei, entwachsen und der Baum, eine Birke, an dem das Kind aufgehängt worden sei, wäre seit dem Mord immergrün gewesen bzw. für sieben Jahre, bis sie gefällt worden sei. Dazu: Schroubek, Zur Frage der Historizität, S. $182-83$.

50 Paulin, Die schönsten Tiroler Sagen, S. 25-27.
} 
Rinner Kirche überführt worden waren, wurden zur Anbetung nun in das neu errichtete Gotteshaus nach Judenstein gebracht..$^{51}$

\subsection{Hippolyt Guarinoni - Schöpfer des Andreas-Kultes}

In den folgenden Jahrhunderten verstärkten sich Anbetung und Kult. Mittlerweile gilt es allerdings als bewiesen, dass die Legende, so wie Guarinoni sie etablierte, fiktiv ist. Der wohl triftigste Anhaltspunkt dafür ist, dass es, wie weiter unten noch aufgezeigt werden wird, kaum archivalische Bestände zum Fall Andreas Oxner von Rinn gibt - weder Gerichtsakten noch Aufzeichnungen der Pfarreien Ampass oder Wilten o. Ä. Erste Belege für diesen Ritualmord sind erst für das 17. Jahrhundert mit den Handschriften Hippolyt Guarinonis greifbar. Die "Urfassung” der Anderl-Erzählung Guarinonis entstand in den Jahren 1620-1622 und ist in der überarbeiteten Form von $1651^{52} \mathrm{im}$ Wiltener Stiftsarchiv einsehbar. ${ }^{53}$ Die Gründe, weshalb Guarinoni die Legende erfand, liegen in seiner Biographie und in den historischen Rahmenbedingungen.

Hippolyt (ital.: Ippolito) Guarinoni wurde am 18. November 1571 in Trient als unehelicher Spross einer einflussreichen, ursprünglich aus Mailand stammenden Arztfamilie geboren. ${ }^{54}$ Guarinoni verbrachte den Großteil seiner Kindheit in seiner Geburtsstadt, wo auch die Legende des Simon von Trient spielte. Es ist anzunehmen, dass ihm daher die Erzählung geläufig war. Die Wahrnehmung derselben wurde aber sicherlich durch die 1588 in Trient stattfindenden Feierlichkeiten zur Aufnahme Simons in das „Martyrologium Romanum ${ }^{455}$ und zur einhergehenden offiziellen Kulterlaubnis verstärkt. ${ }^{56}$

Im Alter von neun Jahren wurde Hippolyt Guarinoni von seinem Vater, der Leibarzt von Kaiser Maximilian II. und danach Kaiser Rudolfs II. war, zuerst nach Wien und dann nach Böhmen mitgenommen. Dort besuchte er in Prag von 1583-1593 die Jesuitenschule. Die Societas Jesu, die sich damals besonders in Belangen der Bildung ${ }^{57}$ und Gegenreformation ${ }^{58}$ engagierte, sollte ein Leben lang eine Ankerfunktion für Guarinoni bleiben. Nach Beendigung der Schule brach der Trentiner 1594 nach Padua auf, um dort Medizin und Kunst zu studieren. Er promovierte 1598 in beiden Fächern. Danach bekleidete er als Mediziner mehrere Posten in Tirol. So fungierte er ab 1598 als Stiftsarzt des königlichen Haller Damenstiftes. Drei Jahre später wurde er zum Stadt- und Salinenarzt der Salzstadt erhoben. Ab 1604 ordinierte er zusätzlich noch als Gewerkenarzt in Schwaz und ab 1607 war er Leibarzt der beiden im Damenstift lebenden Erzherzoginnen. Guarinoni war zweimal mit sehr katholischen und angesehenen Frauen verheiratet: mit Charitas Thaler und nach deren Tod ab 1612 mit Helena von Spieß.

51 Josef Gelmi, Die Geschichte der Kirche in Tirol, S. 100.

52 Guarinoni, Begrü[n]dte Historj [...].

53 Schroubek, Zur Frage der Historizität, S. 176.

54 Ebd.

55 Ebd., S. 179.

56 Ebd., S. 176-180.

57 Österreichische Provinz der Gesellschaft Jesu, Geschichte des Ordens, o. A., [http://www.jesuiten.at/der-orden/ geschichte-des-ordens/], eingesehen 7.1.2017; Gelmi, Geschichte der Kirche in Tirol, S. 163-165 und S. 216-218. 
Besonderer Ruhm wurde ihm wegen seiner medizinischen Verdienste in den Jahren 1611/1612 zuteil, als er an der Seite des Innsbrucker Hofarztes Paul Weinhard gegen die „Pest" (eigentlich Flecktyphus) kämpfte. ${ }^{59}$ Er selbst hatte in dieser Zeit als Schutz vor der Seuche eine Stoffreliquie des von ihm verehrten und mittlerweile heiliggesprochenen Jesuiten Petrus Canisius ${ }^{60}$ bei sich, die ihn vor einer Ansteckung beschützen sollte. Im darauffolgenden Jahr 1613 reiste Guarinoni nach Rom, um Heiligengräber zu besuchen, von wo er der Stiftskirche Hall die sterblichen Überreste der Heiligen Vincentia und Lea mitbrachte. ${ }^{61}$

Guarinoni beschäftigte sich über diesen Besuch Roms hinaus generell mit den Themen Religion und Heiligenfrömmigkeit. Beispielsweise übersetzte und verfasste er mehrere Heiligenviten ${ }^{62}$ und Erbauungsschriften, für die er jedoch z. T. vage Quellen wie etwa Wunderberichte verwendete. ${ }^{63}$ Er galt dabei als sehr erfolgreicher "Volksschriftsteller" ${ }^{\prime 64}$, der mit hohen weltlichen und geistlichen Personen in Kontakt stand ${ }^{65}$ und überdies auch als erfolgreicher Bauherr einer Kirche und einiger Kapellen galt. ${ }^{66}$ In religiösen Belangen pflegte er engen Kontakt zu verschiedenen Orden. Neben den Wiltener Prämonstratensern, ${ }^{67}$ den Innsbrucker Kapuzinern (hier v. a. zu seinem "Seelenführer"68 Bruder Tommaso da Bergamo) ${ }^{69}$ und den Serviten verband ihn ein besonders enges Band mit den Haller Jesuiten. ${ }^{70}$ Die gegenreformatorische und volkserzieherische Societas Jesu, in deren Marianischer Kongregation er ebenfalls Mitglied war, stärkte inm in jeglicher Hinsicht den Rücken: Guarinoni propagierte als "Laienjesuit" ${ }^{\prime 71}$ den Barockkatholizismus und wirkte an Wallfahrten, Prozessionen, Reliquienverehrungen, Andachten und dem sogenannten Jesuitentheater mit. ${ }^{72}$ Dieses Schauspiel sollte die breite Masse in moralischen und religiösen Fragen bilden. In Zeiten der Reformation bzw. reformatorischer Ausbrüche diente es auch dazu, die Bevölkerung beim „rechten

59 Verena Konrad, Guarinoni, Hippolyt (Projekt Artisti Italiani Austria der Universität Innsbruck, Leitung: ao. Univ.-Prof. Peter Fidler), März 2009, [https://www.uibk.ac.at/aia/guarinoni_hippolyt.html], eingesehen 4.1.2017.

60 Guarinonis Verehrung des Canisius bedarf im Zeichen der "Vorbildfunktion“ besonderer Beachtung. Canisius galt als strikter Verfechter der Gegenreformation. Ihm wird etwa zugeschrieben, dass Österreich und weite Teile Süd- und Westdeutschlands dem katholischen Glauben treu blieben. Dazu: Gelmi, Geschichte der Kirche in Tirol, S. 166-170; Bernhard Kriegbaum, Petrus Canisius - ein Lebensbild (27. April), in: Jesuiten. Mitteilungen der österreichischen Jesuiten 70 (1997), Heft 1, S. 4-6, [http://www.jesuiten.at/der-orden/heilige-und-selige-jesuiten/ detail/?no_cache=1\&tx_sihistory_pi1\%5Buid\%5D=17], eingesehen 7.1.2017; Rudolf Leeb, Der Streit um den wahren Glauben - Reformation und Gegenreformation in Österreich, in: Herwig Wolfram (Hrsg.), Geschichte des Christentums in Österreich. Von der Spätantike bis zur Gegenwart (Österreichische Geschichte o. A.), Wien 2003, S. 145-279, hier S. 241-243.

61 Schroubek, Zur Frage der Historizität, S. 176-177.

62 Dazu z. B.: Hippolyt Guarinoni, Prälaten Cron Lebens und Der Gewaltigen Thaten deß H. Caroli Borromaei [...], Freiburg i. Br. 1618.

63 Schroubek, Zur Frage der Historizität, S. 176.

64 Ebd., S. 178.

65 Ebd.

66 Konrad, Guarinoni, Hippolyt, eingesehen 4.1.2017.

67 Klaus Brandstätter, Antijüdische Ritualmordvorwürfe in Trient und Tirol. Neuere Forschungen zu Simon von Trient und Andreas von Rinn, in: Historisches Jahrbuch 125 (2005), S. 495-536, hier S. 524.

68 Noflatscher, Jüdisches Leben in Tirol, S. 225.

69 Ebd.

70 Schroubek, Zur Frage der Historizität, S. 177.

71 Wolftraud Schreiber, Simon von Trient \& Anderl von Rinn. Zwei antijüdische Lügenmärchen, in: Paul Preims/Hans Wielander (Hrsg.), Trentino. Ein Lesebuch, o. O. 2013, S. 31-35, hier S. 34.

72 Schroubek, Zur Frage der Historizität, S. 177. 
Glauben" zu halten. ${ }^{73}$ Es ist anzunehmen, dass ihn besonders seine Schulzeit bei den Jesuiten in Prag, die für ihren an Thomas von Aquin angelehnten, gegenreformatorischen Katholizismus bekannt sind, dahingehend prägte. ${ }^{74}$

Der seit Kindertagen mit dem Simon-Kult vertraute Guarinoni wurde 1637 nach Trient berufen, um die sterblichen Überreste des Trentiner Märtyrerknaben im Zuge der Vorbereitungen zur Eröffnung des Reliquienschreins zu obduzieren, zu salben und zu helfen, diese neu einzukleiden. ${ }^{75}$ Dabei stellte er an den Gebeinen 5812 Wunden fest, die von Jüdinnen und Juden stammen sollten und die er in einem Bericht über Simon von Trient 1638 erwähnte. ${ }^{76}$ 1651, drei Jahre vor seinem Tod (er verstarb am 31. Mai 1654 in Hall i. T. ${ }^{77}$, schrieb Guarinoni seine Aufzeichnungen von 1620-22 zum Anderl von Rinn in revidierter Form nieder. ${ }^{78}$

In diesem Zusammenhang ist noch die Frage nach den Gründen seiner Aversion gegen Jüdinnen und Juden, die sich in den Anderl-Schriften, aber auch im Simon-Bericht, wie auch auffallend oft in anderen Dokumenten finden lassen, zu klären. Die Wurzeln dafür liegen einerseits in seiner Kindheit und in seinen Prager Jugendjahren, in denen er mit angeblichen Gräueltaten der "von Gott vermaledeüten Juden"79 in Berührung kam. Schon früh berichtete er z. B. in „Die Grewel der Verwüstung“80 über die unmenschliche und barbarische Bestrafung eines Juden, der angeblich eine Christin geschändet haben soll. Andererseits betont Schroubek, dass Guarinoni als weitgehend aufgeklärt gelten muss, weshalb sich für die/den Geschichtswissenschaftler_in ergibt, dass eine Angstverlagerung - auch Guarinoni als Gebildeter konnte sich die Umweltphänomene u. Ä. noch nicht erklären - vorlag. Statt z. B. Hexen (an deren Existenz er nicht glaubte) schrieb Guarinoni alles Übel daher den Jüdinnen und Juden als „Teufelsdienern", deren Lehre dem Christentum für ihn diametral gegenüberstand, zu. Außerdem ist anzunehmen, dass auch die berufliche Konkurrenz zwischen jüdischen und nichtjüdischen Ärzten dabei eine nicht zu vernachlässigende Rolle im Judenbild Guarinonis spielte, denn jüdische Leibärzte waren, obwohl Jüdinnen und Juden zu jener Zeit generell kein hohes Ansehen bei der Bevölkerung genossen, wegen ihres Wissens um die Heilkunst sehr gefragt. ${ }^{81}$

Demographisch gesehen gab es wenige Jüdinnen und Juden in Tirol, speziell am Land. Trotz einer dünnen Besiedelung von Jüdinnen und Juden war ab etwa der zweiten

73 Österreichische Provinz der Gesellschaft Jesu, Geschichte des Ordens, eingesehen 7.1.2017.

74 Jung, Reformation und Konfessionelles Zeitalter, S. 187-188.

75 Rudolf Palme, Zur spätmittelalterlichen und frühneuzeitlichen Sozial- und Rechtsgeschichte der Juden in Tirol, in: Alfred Ebenbauer/Klaus Zatloukal (Hrsg.), Die Juden in ihrer mittelalterlichen Umwelt, Köln-Wien 1991, S. 183203, hier S. 196.

76 Brandstätter, Antijüdische Ritualmordvorwürfe in Trient und Tirol, S. 521.

77 Konrad, Guarinoni, Hippolyt, eingesehen 4.1.2017.

78 Brandstätter, Antijüdische Ritualmordvorwürfe in Trient und Tirol, S. 521.

79 Guarinoni, Begrü[n]dte Historj [...], S. 33.

80 Hippolyt Guarinoni, Die Grewel der Verwüstung Menschlichen Geschlechts. Ingolstadt 1610, S. 1130, zit. n. Jürgen Bücking, Kultur und Gesellschaft in Tirol um 1600. Des H. Guarinonius',Grewel der Verwüstung Menschlichen Geschlechts' (1610) als kulturgeschichtliche Quelle des frühen 17. Jahrhunderts (Historische Studien 401), Lübeck-Hamburg 1968, S. 51.

81 Schroubek, Zur Frage der Historizität, S. 179-180. 
Hälfte des 16. Jahrhunderts im Rahmen der Konfessionalisierung der Altkirche eine Judenfeindschaft gefördert worden. Guarinoni, der "ein ,Produkt' der katholischen Reform und Gegenreformation war" ${ }^{\prime \prime 2}$, hegte, wie beschrieben, auch Kontakt zu wichtigen geistlichen Persönlichkeiten, die eine antijudaistische Einstellung vertraten, wie etwa zum in Innsbruck lebenden Kapuzinerbruder Tommaso da Bergamo. ${ }^{83}$

Diese kurzen Schlaglichter auf Guarinonis Leben und Werk ergeben das Bild einer ambivalent denkenden und handelnden Persönlichkeit. Einerseits war Guarinoni ein umfassend gebildeter Humanist, forschender Mediziner und erfolgreicher Bauherr, andererseits ein Hagiograph, der vage Quellen für seine Arbeiten verwendete und ein abergläubischer und gegenreformatorischer Barockkatholizist, der neben Lutheranern_innen auch Jüdinnen und Juden hasste. Er war ein mitunter aktionistischer "Tausendsassa", der, wie eine zeitgenössische Quelle belegt, dadurch nicht immer angenehm auffiel: „Mein Gott, was hebt der guete Doktor Guarinoni nit [alles] an. Wäre wol besser, er bliebe bey seiner Facultät." ${ }^{\prime 4}$

\subsection{Hippolyt Guarinonis Konstruktion der Legende}

Antwort darauf, wie das Rinner "Ritualmordmärchen“ entstanden ist, gibt Guarinoni selbst in seiner Handschrift von 1651. ${ }^{85}$ Dort schreibt er, dass er am Palmsonntag 1619 seiner Familie von dem Fall des Simon von Trient erzählt habe. Daraufhin sei seiner zweiten Gattin, Helena von Spieß, eingefallen, dass sie von „[i] hrer fruom Mutter gehördt, dz dergleichen Jüdische vnthatt negst ober der Stett Hall beÿ dem Dorff Rinn, vor villen Jahren fürüber gangen sein solle".86 Dieser Knabe wäre vor sehr langer Zeit im Tiroler Ort Rinn von jüdischen Kaufleuten zu Tode gequält worden. ${ }^{87}$

In Folge dieser Erzählung habe Guarinoni selbst begonnen, diesbezüglich Nachforschungen anzustellen, da ihm „ain solche begür, diß wunder in Erfahrung zu bringen angefalten, dz er khein ferren Rhu oder Rasst gehabt, biß er (vngeacht des noch eysigen [...] wegs) dahin khumben" ${ }^{\prime 8}$ war. Das erste Indiz, auf das er gestoßen sei, wären die "Gebäin deß hailligen Martÿrers" 89 gewesen, die er in der Rinner Kirche gefunden habe. Eine anschließende Archivrecherche sei dagegen erfolglos geblieben, obwohl ihm "getreue andechtige Gehülffen"90 wie die Erzherzogin Eleonore und Maria Christierna und viele andere Personen hohen Standes bei der Suche nach Akten halfen. Da die Archivrecherche erfolglos blieb, weil - so Guarinonis Erklärung - diese Gräueltaten so schrecklich gewesen seien, dass niemand sie habe aufzeichnen wollen - begann

82 Noflatscher, Jüdisches Leben in Tirol, S. 220.

83 Ebd.

84 Franz Grass, Dr. Hippolytus Guarinonius zu Hoffberg und Volderthurn 1571-1654, in: Raimund Klebelsberg (Hrsg.), Hippolytus Guarinonius (1571-1654). Zur 300. Wiederkehr seines Todestages (Schlern-Schriften 126), Innsbruck 1954, S.9-17, hier S. 16.

85 Guarinoni, Begrü[n]dte Historj [...].

86 Ebd., S. 8.

87 Schroubek, Zur Frage der Historizität, S. 181.

88 Guarinoni, Begrü[n]dte Historj [... ], S. 8.

89 Ebd

90 Ignatius Zach, Ausführliche Beschreibung der Marter Eines heiligen und unschuldigen Kinds Andreae von Rinn in Tyrol und Bistumb Brixen [...], Augsburg 1724, S. 246; Guarinoni, Begrü[n]dte Historj [...], S. 7. 
der Haller, die Bewohner_innen Rinns wegen der Sache zu befragen. Diese wären ihm aber keine große Hilfe gewesen, denn es habe „über alles vleissiges nachfregen Niemendt unter denn Inwohnern, des Namens [Anderl] mer gedacht, oder wissn wellen".91 Deshalb habe er große Mühe und Beharrlichkeit aufwenden müssen, um endlich den Namen des Märtyrerkindes herausfinden zu können. Diesen erfuhr er nach all den vielen Befragungen schließlich durch die Auskunft des ortsansässigen „Mössners, ain Man beÿ 60 Jahrn, welcher nach langen besünnen, in der Kirchen negst beÿ Haillign Martyres Gwöbl, geantwort, Er vermain (së̈ ihm anderst recht), das Khindt hab Anderle gehaissen". 92 Darauf hätten sich auch die anderen „Inwohner hernach widerumb Erÿnert".93

Weitere Details wie die Namen der Eltern Andreas' fand er durch Zufall heraus, als er den Bürgermeister Hueber nach der Ostermesse in Volders traf. Dieser kannte eine gewisse Elisabeth, die eine brennende Anhängerin Anderls sei und die Namen der Eltern, Simon und Maria Oxner, kenne. Sofort stellte Guarinoni nun die Zusammenhänge zwischen Simon von Trient und Anderl von Rinn her: Beide Mütter der Märtyrerkinder heißen Maria, Simons Vater Andrea und Anderls Vater Simon. Die "Suche" nach dem genauen Datum, an dem Anderl getötet worden sei, gestaltete sich da schon schwieriger. Dieses sei Guarinoni nämlich angeblich in der Nacht auf Allerheiligen 1621 in einem Traum ${ }^{94}$ erschienen und steht, wie Schroubek bei seinen Ausführungen zur Historizität vermerkt, wie auch das Sterbedatum Anderls in direktem Zusammenhang mit den Daten um die Trienter Ritualmordtat. ${ }^{95}$ Ziel war es, einen Kult ganz nach dem Vorbild Simon von Trients zu schaffen, der bisher, so Guarinoni, nur aufgrund eines Höllenwerks ausgeblieben sein konnte. ${ }^{96}$

Die überaus umfangreichen Parallelen zwischen der Legende des Simon von Trient und jener des Andreas von Rinn lassen klar erkennen, dass Guarinoni die tirolische Märtyrerlegende auf Basis des florierenden Trienter Kultes aufstellte. Als ein Motiv für diese Erfindung der Anderl-Legende seitens Guarinonis kann eine Art "territorial eingegrenztes Vaterlandsgefüh|"97 gelten, das dem humanistisch geprägten Zeitgeist des 17. Jahrhunderts entsprach. ${ }^{98}$ Auch der Fund einer verstümmelten Kinderleiche, die den Ausgang für eine eventuell bereits früher anzusetzende Legendenbildung markiert und auf die der Arzt aufbaute, ist nach Schroubek nicht undenkbar. ${ }^{99}$ In diesem Sinne wollte der Haller Stadtarzt in Zeiten religiöser und politischer Turbulenzen den Kult um das „vergessene Tiroler Märtyrerkind“ wieder beleben. ${ }^{100}$ Dabei erfuhr er nicht nur von den Wiltener Prämonstratensern (seine zweite Frau stand in Kontakt mit

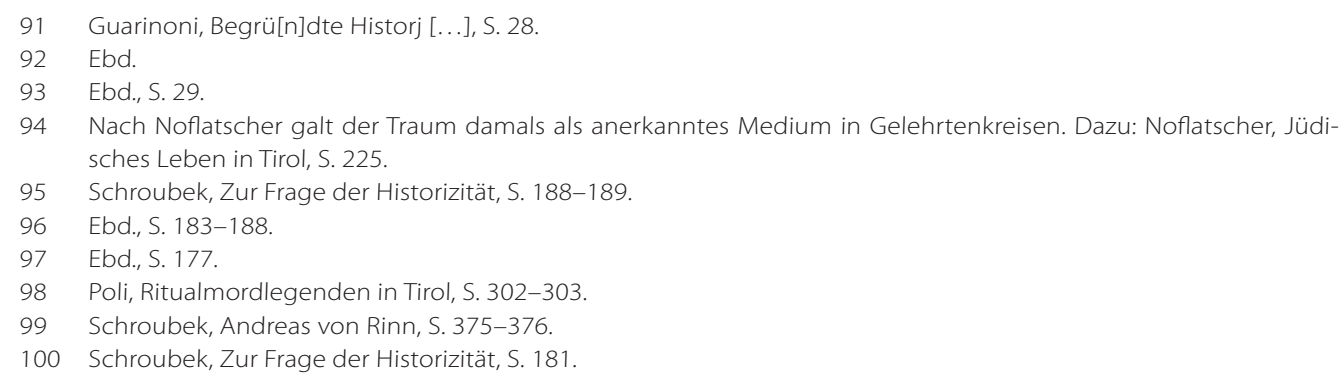


ihnen), ${ }^{101}$ die sich für die rasche Etablierung einer Wallfahrt einsetzten, von Anfang an Unterstützung, sondern vor allem von den gegenreformatorischen Jesuiten. ${ }^{102}$

Basis für die Kultentstehung war also die Erzählung Hippolyt Guarinonis, die durch einen enormen Detailreichtum des Geschilderten einen hohen Grad an Historizität vorgaukelt. Obwohl nach dem Stadtarzt selbst „diße Erbërmbliche tragedi [...] niambandt, als Gott"103 mitverfolgt habe, hielt Guarinoni neben exakten Beschreibungen des Hergangs der gesamten Begebenheit (Vor-, Haupt- und Nachgeschichte samt Wunderberichten) für die Legende wichtige Orte auch zeichnerisch fest. Diese visuelle "Verortung" machte die Legende für jedermann noch greifbarer und glaubhafter. Zur Personalisierung und Legitimation der Geschehnisse der Legende fügte er Rinner Dorfbewohner_innen mit vertrauenserweckenden, damals für Rinn üblichen Namen hinzu, ebenso wie historisch relevante Personen wie z. B. Kaiser Maximilian I. ${ }^{104}$

Bei der Forschung nach dem Wahrheitsgehalt der Vorgeschichte des Anderl-Kultes entdeckte der Geschichtswissenschaftler Heinz Noflatscher eine Lücke in der Berücksichtigung von Quellen - Visitationsberichte - die bisher nicht in die Forschung mit einbezogen wurden. Diese Berichte, sie setzten etwa um 1570 für Rinn und die umliegenden Nachbardörfer ein, werfen ein neues Licht auf die Sache. Aus ihnen geht hervor, dass es bereits 1612 einen Kult um ein verstorbenes, vielleicht sogar getötetes Kind gab. Dieser reicht, wie Noflatscher und vor ihm bereits Schroubek ${ }^{105}$ vermutete, wahrscheinlich sogar mindestens bis ins frühe 16. Jahrhundert zurück, also in eine Zeit, die noch lange vor Guarinonis Ausführungen lag.

Weshalb erinnerte sich dann aber niemand an einen derartigen Kult bei Guarinonis Befragungen? Auch dies lässt sich nach Noflatscher anhand der Visitationsberichte erklären: Die Einwohner_innen Rinns und Umgebung hegten Misstrauen gegenüber allem, was von „außen“ und von herrschaftlicher Seite (politisch und religiös) an sie herantrat. Dazu gehörten beispielsweise Innsbruck als politisches Zentrum oder im klerikalen Bereich die Stadt Hall sowie die in Tirol jungen Orden (z. B. Jesuiten). Guarinoni vereinigte für sie diese Misstrauensfaktoren, da er ein „Mann mit besten Beziehungen zum katholischen Hof sowie zum Hof- und neuen Ordensklerus"106 war. Auch durch Betrachten bisher bereits bekannter und durch Komplettierung neu erschlossener Quellen und Querverbindungen, die sich daraus ergeben, kristallisiert sich zusehends heraus, dass Guarinoni durchaus der Begründer und treibende Motor des für uns heute noch greifbaren Anderl-Kultes ist - jedoch aber, der aktuellen Quellenlage nach, nicht sein Erfinder. ${ }^{107}$

101 Noflatscher, Jüdisches Leben in Tirol, S. 227.

102 Poli, Ritualmordlegenden in Tirol, S. 302-303.

103 Guarinoni, Begrü[n]dte Historj [...], S. 75.

104 Schroubek, Zur Frage der Historizität, S. 189-190.

105 Brandstätter, Antijüdische Ritualmordvorwürfe in Trient und Tirol, S. 521-524; Schroubek, Andreas von Rinn, S. 373-376.

106 Noflatscher, Jüdisches Leben in Tirol, S. 224.

107 Ebd., S. 222-246. 


\section{Der Andreas-Kult}

„Dienen die Schriften der Bollandisten ausschließlich der Unterrichtung der Kleriker [...], so wird das Legenden-,Wissen' der einfachen Gläubigen mit Hilfe anderer Medien weitergetragen. Neben der Überlieferung innerhalb der (Groß-) Familie sind [...] Sondergebete, Litaneien, Kirchenlieder, Predigt, Christenlehre [...] Erbauungs- und religiöse Unterhaltungsschriften, didaktische Volks- und Jugendbücher, [...] [nicht zu unterschätzen] - sie alle verankerten, gewissermaßen nebenher, aber nachhaltig, das,Wissen' um die, jüdischen Religionsfrevel', denen das betreffende, heilige Kind' seine Märtyrerpalme verdankte. Vor allem aber garantierte das Weiterleben solcher örtlicher Traditionen, auch unter nicht mehr so günstigen Zeitumständen, die Vorzeigbarkeit von Reliquien und anderen dinglichen,Bezeugungen' der passio des jeweiligen Kinderheiligen." ${ }^{108}$

Die meisten der von Schroubek identifizierten Tradierungsmedien von Ritualmordlegenden sind auch in der Kultbildung des Anderl von Rinn zu beobachten.

\subsection{Wallfahrtskirche und Pilgertum}

In der unsteten Zeit rund um den Dreißigjährigen Krieg, der Gegenreformation und katholischen Erneuerung ${ }^{109}$ machte sich im Barock eine neue Art der Volksfrömmigkeit breit (in der Tiroler Gegend setzte die Aufklärung erst verspätet ein). ${ }^{110}$ Sie war gezeichnet durch einen enormen Aufschwung von Religiosität und Frömmigkeitsformen, die „aus Christen erst Katholiken“111 machte. In den Mittelpunkt rückten neben gesteigertem Legenden- und Heiligenglauben auch Eucharistie, Prozessionen, Bußumzüge, Volksschauspiele und Wallfahrten. ${ }^{112}$

Wallfahrten, so Gelmi, entstanden aus „der Idee heraus, dass Gott dem heilsbedürftigen Menschen an bestimmten Orten besonders nahe ist", weshalb sie "vermutlich so alt ist wie die Menschheit selbst".113 Im Falle Rinns folgte die Wallfahrt zudem „einem geläufigen Muster des gegenreformatorischen Wettstreits kirchlicher Institutionen um Kultstätten und Wallfahrtsorte, in denen sich die populären Formen der tridentinischen Frömmigkeit durchführen ließen".114 In diesem Sinne wurde in Tirol die JudensteinWallfahrt besonders durch die Prämonstratenser und das Damenstift Hall propagiert.115

Den Grundstein für diese florierende Wallfahrt legte Guarinoni selbst: Das Anderl von Rinn galt wegen diverser Mirakeln, die an seinem Grab geschehen sein sollen, besonders in Tirol, aber auch transregional (Südbayern und Trient) als Fürsprecher bei Gott. An jenem Ort, an der die Marter vonstatten gegangen sein soll („Judenstein“), wurde ein

\footnotetext{
108 Schroubek, Zur Tradierung und Diffusion, S. 22-23.

109 Gelmi, Geschichte der Kirche in Tirol, S. 163-179.

110 Noflatscher, Jüdisches Leben in Tirol, S. 218-219.

111 Gelmi, Geschichte der Kirche in Tirol, S. 192.

112 Ebd., S. 192-195.

113 Ebd., S. 118.

114 Holzmann, Vier Ritualmordlegenden im Vergleich, S. 81.

115 Ebd.
} 
Gedenkkreuz errichtet. Als, nach Guarinoni, Kaiser Maximilian I. 1507 dorthin gepilgert war, beschloss dieser, dort eine Kirche zu Ehren des "Märtyrers" erbauen zu lassen. ${ }^{116}$ Das Geld, welches der Kaiser dafür bereitgestellt habe, berichtet der Stadtarzt weiter, sei einem Bauern, Jakob Haan, in Verwahrung gegeben worden. Da dieser jedoch das Geld veruntreut habe, habe damals der Bau der Kirche unterlassen werden müssen. ${ }^{117}$

Die Idee dazu wurde jedoch nicht komplett verworfen, sondern unter Guarinoni 1642 wieder reaktiviert, konnte aber erst nach seinem Tod 1654 verwirklicht werden. Der Haller Stiftsarzt hatte das für den Bau vorgesehene Grundstück angeblich selbst mit dem Pfarrer von Ampass 1649 vermessen und in seinem Testament vermerkt, dass dreihundert Gulden für das Vorhaben aufgewendet werden sollten. Des Weiteren wurde das Bauunterfangen durch Spenden, die zwischen 1659 und 1661 in Tirol gesammelt wurden, finanziert: Erzherzog Ferdinand Karl (Tiroler Landesfürst 1646-1662) und seine Frau Anna Medici spendeten insgesamt vierhundert Gulden sowie Grund und Baumaterial für das Mesnerhaus. Der Bischof von Brixen stellte überhaupt nur fünfzig Gulden, jener von Augsburg, Trient und Chur zwanzig, die Landstände dreißig Gulden etc. zur Verfügung. Insgesamt kam so eine Summe von 1440 Gulden zustande, mit der das Gotteshaus finanziert werden konnte. ${ }^{118}$

1660/61 war Baubeginn und bereits wenige Jahre später 1670 konnte der Sakralbau, wahrscheinlich unter Gallus Appeller, vollendet werden. ${ }^{119} 1678$ wurde die Kirche am Judenstein als Filialkirche Rinns im ersten Patronat den unschuldigen Kindern und im zweiten Patronat dem "seligen“ Andreas von Rinn ${ }^{120}$ geweiht. Die Weihe nahm Paulinus Mayr, Fürstbischof zu Brixen, vor. ${ }^{121}$

Der Bau besticht architektonisch durch einen rechteckigen Grundriss $(23 \mathrm{~m} \times 7,7 \mathrm{~m})^{122}$ mit eingezogenem Chor und einem Nordturm, der im Aussehen an jenen Stift Wiltens erinnert. Das nach Osten hin ausgerichtete dreijochige Langhaus wird überdacht von einem Stichkappentonnengewölbe, das auf Pfeilern ruht. ${ }^{123}$ Pilaster, ein rundbogiger Triumphbogen und eine Westempore ohne Stützen gliedern den Innenraum der Kirche. Seit 1730/40 zieren zudem Rokokostuckaturen (Blüten- und Bandlwerk) ${ }^{124}$ in Pastelltönen die Gewölbe des Chors und des Triumphbogens. Die Stuckverzierung bildet an der Langhausdecke Bildfelder, die mit Fresken des Künstlers Josef Ignatz Mildorfer versehen sind. ${ }^{125}$

116 Teilweise ist in der Literatur auch zu lesen, dass unter Maximilian I. ein Kapellenbau am Ort der heutigen Kirche in Judenstein stattgefunden habe. Dazu: Kapferer-Prinoth/Kapferer, Rinn:Wallfahrtskirche, S. 146; Öttl/Franckenstein u. a., Rinn: Ehem. Wallfahrtskirche, S. 656.

117 Holzmann, Vier Ritualmordlegenden im Vergleich, S. 82.

118 Noflatscher, Jüdisches Leben in Tirol, S. 243.

119 Öttl/Franckenstein u. a., Rinn: Ehem. Wallfahrtskirche, S. 656.

120 Ebd., S. 655.

121 Gelmi, Geschichte der Kirche in Tirol, S. 100

122 Florian N. Schomers, Wallfahrtskirche Mariae Heimsuchung, Judenstein, O. O. 2009, S. 4.

123 Kapferer-Prinoth/Kapferer, Rinn: Wallfahrtskirche, S. 146.

124 Schomers, Wallfahrtskirche Mariae Heimsuchung, S. 5.

125 Kapferer-Prinoth/Kapferer, Rinn: Wallfahrtskirche, S.146. 
Diese Bildfelder zeigen, heute teilweise abgedeckt, von Westen nach Osten Szenen aus der Andreas-Legende: den Verkauf von Andreas durch seinen Taufpaten, die Ermordung Anderls durch die Juden, Maria Oxner in ihrer bösen Vorahnung auf den Ambraser Feldern inkl. der drei Blutstropfen und die Glorie des "Märtyrers" im Himmelreich. Nördlich und südlich des vierten Freskos, welches den Chorraum schmückt, befinden sich in den Kartuschen Camaieu-Fresken. ${ }^{126}$

Besonders erwähnenswerte Einrichtungsgegenstände sind die Kanzel aus Stuckmarmor (Mitte 18. Jahrhundert) und der linke Seitenaltar, der, zwischen Säulen gefasst, eine Kopie der "Maria vom Guten Rat" zeigt, die barocke Orgel, ${ }^{127}$ außerdem eine Statue des HI. Nepomuk (Stefan Föger) und die Kreuzwegbilder von Johann Georg Dominikus Grasmair. $^{128}$

Noch bevor der Kult um Andreas von Rinn 1988/89 (der Prozess dauerte von 19851988/89) ${ }^{129}$ auf Betreiben des Altbischofs Reinhold Stecher abgeschafft wurde ${ }^{130}$ und die Kirche der Mariä Heimsuchung geweiht wurde, mussten einige Anderl-Kult-Gegenstände im Rahmen der Beschlüsse des Zweiten Vatikanischen Konzils ${ }^{131}$ weichen. ${ }^{132}$

Als erste Veränderungen wurden 1961 eine Legendentafel, die von Johann Georg Dominikus Grasmair 1730 geschaffen worden war, entfernt und das Anderl-Gemälde ${ }^{133}$ hinter dem „Judenstein“ abgedeckt. Ebenso wurden im selben Jahr die türkisch gewandeten Juden der barocken Figurengruppe von Franz Xaver Nissl, die ursprünglich auf dem Platz des rechten Seitenaltars auf dem „Judenstein" exponiert waren, abgebaut. ${ }^{134}$ Das Anderl, welches auch dazu gehörte, folgte 1988/89. Stattdessen wurde ebenfalls 1988/89 die „Ölberggruppe" (Jesus und schlafende Jünger) auf dem „Judenstein“ aufgestellt. Außerdem wurde im selben Jahr das Deckenfresko Mildendorfers, welche die Marter Anderls zeigte, durch eine Spanplatte überdeckt. Darauf neu zu sehen ist nun das von Wolfram Köberl in barocker Tradition gemalte Bild "Jesus als göttlicher Kinderfreund". Des Weiteren geht die Neugestaltung des Hochaltarbildes 1989, das nun die Heimsuchung Mariens zeigt, ebenfalls auf Köberl zurück. ${ }^{135}$ Zuvor waren am Hochaltar die prunkvoll gekleideten und mit Glassteinen gefassten Gebeine des Anderl in einem wertvollen Glasschrein ausgestellt worden. ${ }^{136}$

Besonders interessant ist, was mit den Gebeinen Andreas' passierte, da sie der eigentliche Grund für den Bau der Kirche als adäquate letzte Ruhestätte des Märtyrers waren und die Wallfahrt begründeten. ${ }^{137}$ Die Skelettfragmente Andreas' wurden 1475 zuerst

126 Schomers, Wallfahrtskirche Mariae Heimsuchung, S. 5-6.

127 Ebd., S. 13.

128 Kapferer-Prinoth/Kapferer, Rinn: Wallfahrtskirche, S.147

129 Holzmann, Vier Ritualmordlegenden im Vergleich, S. 85-87.

130 Fresacher, Gedächtnis im Wandel, S. 345-436.

131 Die Kirche distanzierte sich in diesem Konzil, das von 1961-1965 dauerte, u. a. von jeglicher antijüdischen Ritualmordtradition. Dazu: Holzmann, Vier Ritualmordlegenden im Vergleich, S. 83-84.

132 Kapferer-Prinoth/Kapferer, Rinn: Wallfahrtskirche, S.146.

133 Öttl/Franckenstein u. a., Rinn: Ehem. Wallfahrtskirche, S. 656.

134 Fresacher, Anderl von Rinn, S. 51.

135 Kapferer-Prinoth/Kapferer, Rinn: Wallfahrtskirche, S.146.

136 Schomers, Wallfahrtskirche Mariae Heimsuchung, S. 9.

137 Zach, Ausführliche Beschreibung der Marter [...], S. 222 
vom Friedhof in Rinn in die Rinner Pfarrkirche übertragen. ${ }^{138}$ Dort wurden sie hinter dem Hochaltar aufbewahrt, bis zur Fertigstellung der Kirche am Judenstein 1671, worauf sie dorthin transferiert und rechts neben dem Hochaltar in einem Schrein exponiert wurden. ${ }^{139} 1743$ wurden sie dort entnommen, um 1744, nachdem sie von den Schwazer Gräfinnen zu Tannenberg ${ }^{140}$ ein Prunkkleid erhalten hatten, in neuem Glanz wieder am selben Ort ausgestellt zu werden. ${ }^{141}$

Aufgrund der dortigen Ausstellung der Gebeine kam es bereits bald zu ersten Wallfahrten. Die erste dokumentierte unternahm 1684 Erzherzogin Eleonore, die dem Anderl eine "heilsvermittelnde Legitimation"142 zusprach, denn nach ihrem Verständnis war ihr Sohn durch die Fürsprache Andreas' von seinen Gehbeschwerden geheilt worden. Als Dank opferte sie die erste Votivgabe, ein Paar silberne Schühlein. ${ }^{143}$ In Folge baten die meisten Pilger_innen um die Fürsprache des Märtyrerleins bei Leiden ihrer Kinder und hinterließen nicht selten als Dank Votivtafeln. ${ }^{144}$ Die Darstellungen von Andreas auf solchen Tafelbildern unterscheiden sich leicht, wobei ein Darstellungstypus klar dominiert: Zumeist wird der "Märtyrer" in einem langen, rotgefärbten Kleid mit weißer Latzschürze und weißer Halskrause alleine oder zusammen mit anderen Heiligen auf einer Wolke schwebend dargestellt. Das Rot des Gewandes soll dabei das geflossene Blut symbolisieren, die weiße Krause und die Schürze die Unschuld des Kindes. In seiner Rechten hält es das typische Märtyrerattribut, einen Palmzweig. ${ }^{145}$

Die Anzahl der Votivtafeln, die in der Kirche in Judenstein hingen, belief sich auf rund siebzig Stück. Daneben wurden auch an anderen Wallfahrtsorten wie z. B. in Absam, Tafeln, die das Anderl beinhalten, gespendet - mindestens zwölf Tafeln. ${ }^{146}$ Dabei gilt, dass sowohl in Judenstein als auch in Absam die meisten derartigen Votivbilder im 19. Jahrhundert gespendet wurden. ${ }^{147}$ Neben Bildern wurden auch noch andere Devotionalien wie Wachskindchen geopfert. ${ }^{148}$

Im Zuge des Kultverbots wurden die Reliquien schließlich in einem verlöteten Metall$\operatorname{sarg}^{149}$ in die südliche Kirchenwand eingemauert. Heute erinnert an diesem Platz eine

138 Holzmann, Vier Ritualmordlegenden im Vergleich, S. 81.

139 Noflatscher, Jüdisches Leben in Tirol, S. 245.

140 Schroubek schreibt, dass es sich um Elisabeth Helena und Maria Anna von Tannenberg handelt. Dazu: Schroubek, Zur Verehrungsgeschichte des Andreas von Rinn, S. 3847.

141 Holzmann, Vier Ritualmordlegenden im Vergleich, S. 82.

142 Noflatscher, Jüdisches Leben in Tirol, S. 243.

143 Ebd.

144 Adrian Kembter berichtet in seiner Publikation zum Anderl von Rinn von Votivtafeln und Devotionalien, die gespendet wurden und den damit geschehenen „Wundern“. Dazu: Adrian Kembter, Acta pro veritate martyrii corporis et cultus publici B. Andreae Rinnesis pueruli anno MCCCCLXII. die 12. julii a judaeis in odium fidei occisi [...], Innsbruck 1745, S. 267-271, zit. n. Nikolaus Christian Kogler, Votivbilder aus dem östlichem Nordtirol I, phil. Diss. Innsbruck 1962, S. 25.

145 Nikolaus Christian Kogler, Votivbilder aus dem östlichem Nordtirol II, phil. Diss. Innsbruck 1962, S. 24.

146 Ebd., S. 4-43.

147 Schroubek bestätigt Koglers Ausführungen mit kleinen Abweichungen, weshalb bei der Angabe der Anzahl der Votivtafeln ein Mittelwert angegeben wird. Dazu: Schroubek, Zur Verehrungsgeschichte des Andreas von Rinn, S. 3850.

148 Kogler, Votivtbilder I, S. 24-25.

149 Gottfried Melzer, Das selige Kind Andreas von Rinn. Ein wahrer Märtyrer der katholischen Kirche, Durach 1989, S. 106. 
Marmortafel mit Inschrift auf der Innenseite der Kirche noch daran. Eine zweite Tafel gedenkt der Verbrechen gegen die Jüdinnen und Juden und befindet sich neben der Ölberggruppe. ${ }^{150}$

\subsection{Predigten, Prozessionen, Andachten und Gebete}

Gemäß einer fast schon fanatischen Heiligen- und Märtyrerverehrung, die neben einem übersteigerten Wunderglauben ein weiteres Kennzeichen der barocken Volksfrömmigkeit war, wurden unterschiedliche Gedenkformen zelebriert. ${ }^{151}$

Als besonders volkserzieherisch und die Masse beeinflussend können dabei Predigten gewertet werden. Nach Schroubek griffen die lokalen Priester in ihren Predigten sehr schnell den Anderl-Fall auf. ${ }^{152}$ Die Kultverbreitung potenzierte sich sozusagen nicht nur von Klerus zu Gläubigen, sondern auch durch Wechselwirkungen zwischen „oben“ und "unten". 153 Daneben wurde die Predigt als Werbemedium auch wirksam außerhalb des Kerntiroler Raumes verwendet. Dies belegen bisher unbekannte Predigten aus dem 18. Jahrhundert, auf die Franz M. Eybl unlängst hinwies.

So hielt z. B. der Jesuit Anton Staudinger 1743 vor der Tiroler Landsmannschaft in der Wiener Jesuitenkirche eine Ansprache. In dieser Rede zum Rinner "Märtyrerlein“, in der auch Simon von Trient vorkam, erzeugte er Angst bei seinen Zuhörer_innen durch das Schüren andijüdischer Vorurteile. Daher, und auch aus rhetorischen Gründen (amplificatio) ${ }^{154}$, war er darauf bedacht, möglichst viele Einzelheiten bei der Schilderung der Mordbeschreibung zu nennen. Es ging ihm darum, bei den Zuhörer_innen ein möglichst gut vorstellbares und gefühlsbetontes Bild der Erzählung zu schaffen. ${ }^{155}$

„Die Blutgierigen Juden eylen mit ihrer Beuth einem Birck=Wäldlein zu: der kleine ANDREAS wird auf einem Stein creuz=weiß ausgestrecket, und gebunden: da dann der Ober=Juden=Pfaff der unmenschlichen metzgerey den Anfang machte. Er schneidet dem unschuldigen Kind an beeden schläffen die $P u l ß=A d e r n$ entzwey; von denen Schläffen wendet er sich zu denen Wängelein, die er mit gleicher Grausamkeit mit tieffen Wunden zerfleischet: hierauf setzt er das Messer an die Brust ANDREÆ, in welche er eine Wunden nach der anderen schneidet; biß er entlich zum Spott des Christenthums nach jüdischer Art die Beschneidung vorgenommen.."156

150 Holzmann, Vier Ritualmordlegenden im Vergleich, S. 85-86.

151 Gelmi, Geschichte der Kirche in Tirol, S. 194-195.

152 Schroubek, Andreas von Rinn, S. 377.

153 Schroubek, Zur Verehrungsgeschichte des Andreas von Rinn, S. 3848.

154 Franz M. Eybl, Das Anderl von Rinn in barocken Predigten, in: Alfred Ebenbauer/Klaus Zatloukal (Hrsg.), Die Juden in ihrer mittelalterlichen Umwelt, Köln-Wien 1991, S. 27-34, hier S. 29.

155 Eybl, Das Anderl von Rinn in barocken Predigten, S. 28

156 Ebd., S. 29. 
Nach Langer gab es mit höchster Wahrscheinlichkeit seit der Approbation des Kultes auch Andreas-Festtagspredigten, die sich jedoch in den Archiven nicht erhalten haben. ${ }^{157}$

Im Sinne des Barockkatholizismus galten neben der Liturgie selbst auch Prozessionen und Andachten als probates Mittel der katholischen Konfessionalisierung. Sie können nach Leeb wie die Wallfahrten als Sozialdisziplinierungsmaßnahmen gesehen werden. ${ }^{158}$

Da es sich zu Beginn der Anderl-Verehrung um einen lokal stark begrenzten Kult handelte, den erst Guarinoni bzw. die katholische Konfessionalisierung förderten, gibt es zwischen 1612 (der quellenmäßigen Erstnennung eines Kultes) und 1651 (Fertigstellung der revidierten Handschrift Guarinonis) keine fundierten Belege für Prozessionen. ${ }^{159}$ Schroubek vermutet jedoch bereits ab mindestens 1620 einen jährlich wiederkehrenden Festumzug am Dreifaltigkeitssonntag zu Ehren der Übertragung der Gebeine Anderls vom Grab in die Rinner Kirche. ${ }^{160}$

Als gesichert gelten Prozessionen erst ab dem 18. Jahrhundert. Auf dem „ProzessionsFixprogramm" standen fortan mehrere Bittgänge: Ab 1744, dem Jahr der Transferierung der neugefassten Gebeine von Schwaz in die Kirche am Judenstein, fand alle fünfzig Jahre eine Prozession statt. ${ }^{161}$ Diese Tradition wurde mittels zwei Tagen dauernden Übertragungsfeierlichkeiten eingeläutet, wobei an jedem Ort, an dem die Prozession auf dem Weg nach Rinn vorbei kam, Andachten abgehalten wurden. Die Prunkprozession war streng choreographiert. Die damit beabsichtigte Verbreitung des Kultes ging voll auf und erreichte in den folgenden Jahren ihren absoluten Zenit. ${ }^{162}$ Ab 1755, dem Jahr der Andreas-Kult-Bestätigung durch Papst Benedikt XIV., fand ein jährlicher Festzug am 12. Juli, dem angeblichen Todestag Andreas', statt. ${ }^{163}$

Daneben wurden von Gemeinden und verschiedenen Bruderschaften Prozessionen organisiert, die nicht unbedingt einem Zyklus unterstanden, sondern bei Bedarf abgehalten wurden. Etwa jene am 4. Oktober 1778, die von der Rosenkranzbruderschaft in Jenbach organisiert wurde und bei der zwei „spanische Edelknaben mit den Marterzeichen des Anderl von Rinn"164 vorweg gingen. ${ }^{165}$

Ebenso erlebten die „außerliturgischen Volksandachten“166 und Bittandachten, die meist von Orden getragen wurden, im 18. Jahrhundert eine Hochblüte. ${ }^{167}$ Sie wurden von der Kirche zur Festigung des Anderl-Kultes angeordnet und durch Schriften wie

\footnotetext{
157 Langer, Zwischen Aggression und Vorurteil, S. 258.

158 Leeb, Der Streit um den wahren Glauben, S. 276-278.

159 Noflatscher, Jüdisches Leben in Tirol, S. 234.

160 Schroubek, Andreas von Rinn, S. 377.

161 Petzoldt, Religion zwischen Sentiment und Protest, S. 177.

162 Schroubek, Zur Verehrungsgeschichte des Andreas von Rinn, S. 3847-3848.

163 Petzoldt, Religion zwischen Sentiment und Protest, S. 176.

164 Gelmi, Geschichte der Kirche in Tirol, S. 195.

165 Ebd.

166 Ebd., S. 201.

167 Ebd.
} 
der „Kurzverfassten Martergeschichte des Heiligen Knaben Andreä von Rinn“,168 die neben der Passion auch Gebete $u$. Ä. enthielt, gefördert. ${ }^{169}$

Nach Langer nahmen eben solche Andachtsgebete, wie allgemein Gebete und im Speziellen Breviergebete, die ab 1754 in Brixen und Rom gedruckt wurden, eine wichtige Rolle in der Verfestigung des Kultes ein. Sie gelten als außerordentlich volkserzieherisch und indoktrinierend. Im Kern wird dabei fast ausnahmslos die Martergeschichte des Anderl nacherzählt: „Es wird von rohen Juden-Händen/ Dein schuldlos Blut dir ausgepreßt,/ Und keine Klag' gen Himmel senden/ Die zugeschnürte Kehl'dich läßt."170

Neben solchen offiziellen Liturgietexten erschienen bereits vorher, ab etwa 1718, verschiedene Litaneien, die für die Heiligenverehrung aus zwei Gründen wichtig waren. Erstens galten die Anrufungen als von der Kirche approbiert und zweitens wurden die Legenden in stark verkürzter Form wiedergegeben, weshalb sie sich besonders gut einprägen ließen. ${ }^{171}$ Beispiele für diesbezügliche Erscheinungen sind die „Litanei zum hl. Anderl"172 sowie das "Gebet am Marterstein" ${ }^{\prime 173}$, beide von 1768.

Auch heute gibt es noch (Gebets-)Bücher zum Anderl von Rinn. Sie wurden beispielsweise gegen Ende des 20. Jahrhunderts von dem durch das Festhalten am AndreasKult negativ bekannt gewordenen und heute suspendierten Kaplan Melzer verfasst bzw. zusammengestellt. ${ }^{174}$ Die Bücher enthalten in der Regel die Anderl-Legende und historische Gebete, teilweise auch Liedtexte. Als Quellen werden dabei neben Quellen aus dem 19. Jahrhundert die Schrift von Ignatius Zach ${ }^{175}$, Adrian Kembter ${ }^{176}$ und jene Guarinonis von $1651^{177}$ angegeben. Leider kann ein für die Arbeit angedachter Vergleich von Gebeten an dieser Stelle nicht erfolgen, da sich in allen drei historischen Schriften auch nach ausführlicher Einsicht im Wiltener Stiftsarchiv unter Mithilfe von Dr. Hannelore Steixner (Archivarin) keine Gebete darin finden ließen. Die Zuordnung der Gebete (zeitlich und urheberrechtlich) aus Melzers Gebetsbüchern, die dahingehend nicht gekennzeichnet sind, ist somit nicht möglich. Nach eingehender Recherche gilt jedoch als gesichert, dass sich die ersten Gebete zum Anderl im Anhang von Guarinonis Schrift "Triumph Cron [...]"178 befinden. Da es durch die Eigenrecherche der

168 Schroubek, Zur Verehrungsgeschichte des Andreas von Rinn, S. 3848

169 Ebd.

170 Anonym, Das heilige unschuldige Kind Andreas von Rinn, von den Juden gemartert im Jahre Christ 1462, in: Anonym (Hrsg.), Heiliger Tyroler-Ehrenglanz, oder Lebensgeschichten heiliger, seliger, gottseliger, frommer und ausgezeichneter Tyroler, Heft 4, Innsbruck 1844, 13-52, hier S. 50 f, zit. n. Michael Langer, Zwischen Vorurteil und Aggression. Zum Judenbild in der deutschsprachigen katholischen Volksbildung des 19. Jahrhunderts (Lernprozeß Christen Juden 9), Freiburg im Breisgau 1994, S. 255.

171 Schroubek, Zur Verehrungsgeschichte des Andreas von Rinn, S. 3851.

172 Langer, Zwischen Vorurteil und Aggression, S. 255.

173 Ebd.

174 Melzer, Das selige Kind Andreas von Rinn, S. 137-150; Gottfried Melzer, Wir beten zum seligen Märtyrerkind Andreas von Rinn. Unschuldiges Kind und Märtyrer. Gebete und Novene zu Ehren des seligen Kindes nebst einem Lebensabriss, Bad Hall 1994. S. 33-87.

175 Zach, Ausführliche Beschreibung der Marter Eines heiligen und unschuldigen Kinds Andreae [...].

176 Kembter, Acta pro veritate martyrii corporis et cultus publici B. Andreae Rinnesis pueruli [...].

177 Guarinoni, Begrü[n]dte Historj [...].

178 Hippolyt Guarinoni, Triumph Cron Marter Vnd Grabschrifft deß Heilig-Vnschuldigen Kindts ANDREAE Von Rinn [...], Innsbruck 1642 . 
Autorin dieser Arbeit und nach Vermerken Langers ${ }^{179}$ offensichtlich in Archiven, wenn auch nicht in den von Melzer genannten Werken, historische Gebete zum Anderl gibt, könnten diese in einer gesonderten Forschungsarbeit eine vergleichende Zusammenstellung erfahren. Den Rahmen dieser Arbeit würde dies jedoch sprengen.

\subsection{Publikationen/Lieder und Volksschauspiele}

Weitere Propagandamedien zur Verfestigung des Kultes waren Publikationen und Lieder, die oft gekoppelt vorkamen. Einige Publikationen waren in Reimform verfasst und teilweise mit populären Melodien hinterlegt.

Drei Jahre nachdem Guarinoni seine unveröffentlichten Aufzeichnungen zur AnderlLegende von 1620-22 nochmals 1651 in überarbeiteter Form, die abermals nicht veröffentlicht wurde, zu Papier gebracht hatte, verstarb er. In der Zeit zwischen diesen beiden Handschriften entstand noch ein weiteres Werk zum Rinner "Märtyrerlein", das 1642 erstmals verlegt wurde und bis 1875 als Vorlage für weitere ergänzende bzw. weiterführende historische und theologische Schriften dienen sollte: „Triumph Cron Marter Vnd Grabschrifft deß Heilig-Vnschuldigen Kindts ANDREAE Von Rinn [...]“.180 Die Legendenerzählung war in Reimform verfasst und mit zwanzig kleinen Stichen versehen. ${ }^{181}$ Sie konnte, wenn auch nicht ganz flüssig, "Gesangsweiß, Im thon des Teuren Ritters vnd Grafen Herrn Niclaus Von Serin"182 gesungen werden. ${ }^{183}$ Der Druck war so bedeutend, dass er bereits 1677 eine zweite Auflage erfuhr und im 18. Jahrhundert, neben handschriftlichen Ausgaben, eine dritte Auflage mit dem Titel "Alt-neu-Historisches Traur und Triumph-Lied Deß durch Judische Grausamkeit ermordeten Kindleins Andreae von Rinn"184, folgte. ${ }^{185}$ In dieser dritten Auflage, die 1729 erschien, erfuhr sie von einem Wiltener Prämonstratenser, wahrscheinlich Casimir Grustner von Grusthof, 186 eine Bearbeitung und Ergänzung der ursprünglich 73 auf 150 leicht zu merkende Strophen, was ihre große Popularität abermals steigerte. ${ }^{187}$ Das Lied galt innerhalb kurzer Zeit als das „verbreitetste und zäheste Tiroler Volkslied“188, wie es vom Volkskundler Anton Dörrer genannt wurde und war besonders bei den ungebildeteren Schichten sehr beliebt. ${ }^{189}$

1661 räumte der Jesuit Georgius Seiser der Anderl-Legende einen beträchtlichen Platz in seinen Ausführungen zum heiligen Romedius ein und verwies darin auf eine räum-

179 Langer, Zwischen Vorurteil und Aggression.

180 Holzmann, Vier Ritualmordlegenden im Vergleich, S. 67.

181 Noflatscher, Jüdisches Leben in Tirol, S. 240-243.

182 Schroubek, Zur Verehrungsgeschichte des Andreas von Rinn, S. 3846.

183 Schroubek, Andreas von Rinn, S. 377.

184 Schroubek, Zur Verehrungsgeschichte des Andreas von Rinn, S. 3847.

185 Ebd., S. 3846.

186 Schroubek meint im Gegensatz zu Langer, der keinen Verfasser nennt, dass es sich um Casimir Grustner von Grusthof handeln könnte. Dazu: Ebd., S. 3847.

187 Michael Langer, „Blutbegier'ge Judenhunde streichen durch dies fromme Land...." Ritualmordwahn und Tiroler Volksfrömmigkeit, in: Diözese Innsbruck (Hrsg.), Judenstein. Legende ohne Ende, Innsbruck o. J., S. 31-62, hier S. 45 .

188 Langer „'Blutbegier'ge Judenhunde streichen durch dies fromme Land....”, S. 45.

189 Ebd. 
liche Nähe der "Heiligen“ Andreas und Simon zu Romedius. ${ }^{190}$ Auch in die Sachliteratur flossen die in Neuauflagen von Guarinonis "Triumph Cron [...]" geschilderten Legendeninhalte ein. So rezipierte etwa Franz Adam von Brandis bereits 1678 die für ihn erinnerungswürdige Geschichte in seiner Landesgeschichte. ${ }^{191}$ Auch Maximilian Graf von Mohr, ebenso wie Brandis ein bedeutender Autor in Bezug auf die Regional- und Landeskunde, nahm die Anderl-Erzählung in seine Betrachtung "Von der Fürstlichen Graffschaft Tyrol" (unveröffentlichtes Manuskript) auf. 1723 schließlich erschien im Zeichen der Verwissenschaftlichung der Theologie ein Eintrag im Rahmen einer enzyklopädischen Zusammenschau aller Heiligen der Jesuiten, den ein Ordensbruder namens Ernst Bidermann vorgenommen hatte. Jedoch waren darin einige Fehler enthalten ( $z$. B. Datum von Anderls Gedenktag). ${ }^{192}$

Neben unbekannteren Autoren gehören zu den bekanntesten Rezipient_innen Guarinonis Ignatius Zach und der Prämonstratenser Chorherr Adrian Kembter. Besonders beliebt waren die Ausführungen von Ignatius Zach ${ }^{193}$ zur Legende, da er sie mit zahlreichen Kupferstichen lebendig gestaltete. Allerdings waren sie dadurch teuer und nur einem potenten Publikumskreis zugänglich. ${ }^{194}$ Das Druckwerk, das weitestgehend die Ausführungen Guarinonis von 1651 wiedergibt und auf Deutsch in Fraktur zu lesen ist, erschien 1724. ${ }^{195}$ Auch Klaus Brandstätter hebt den besonderen Wert von Zachs Publikation hervor, da die enthaltenen Stiche zur Vorlage von populären Bildgeschichten wurden. ${ }^{196}$ Ebenso äußerte sich 1732 der Jesuit Jacob Schmid in seiner Erbauungsschrift „Heiliger Ehren-Glantz"197 zum Anderl. ${ }^{198} 1734$ erwähnte Anton Maximilian Heiß in seiner "Kleine Bauren-Legend Oder Heiliger Hirten und Bauren Leben"199 "Tagelöhner" als Beruf des verstorbenen Vaters von Anderl. Der niedrige Stand des Vaters war der Grund für die Aufnahme der Anderl-Legende in sein Werk. Das Buch, in dem dem Kind Andreas die Seiten 1-28 des vierten Teils eingeräumt wurden, war sehr verbreitet und erlebte noch drei weitere Auflagen in den Jahren 1736, 1750 und 1770 (alle in Augsburg). ${ }^{200}$

Ein weiteres sehr wichtiges Werk zu Andreas von Rinn erschien 1745. Der lateinische Druck ${ }^{201}$ des Chorherren und Kirchenrechtlers Adrian Kembter reflektierte auf Basis eigener Forschung in den ersten beiden Kapiteln zum ersten Mal den offensichtlichen Quellenmangel Guarinonis bei der "Legendenfindung". Dies war in den bisherigen

190 Schroubek, Zur Verehrungsgeschichte des Andreas von Rinn, S. 3846

191 Noflatscher, Jüdisches Leben in Tirol, S. 245.

192 Schroubek, Zur Verehrungsgeschichte des Andreas von Rinn, S. 3846-3847.

193 Zach, Ausführliche Beschreibung der Marter Eines heiligen und unschuldigen Kinds Andreae [...].

194 Ebd.

195 Noflatscher, Jüdisches Leben in Tirol, S. 245

196 Brandstätter, Antijüdische Ritualmordvorwürfe in Trient und Tirol, S. 525.

197 Jacob Schmid, Heiliger Ehren-Glantz der Gefürsteten Graffschaft Tyrol/Das ist: Geschicht/ und Lebens-Verfassung Aller der Jenigen Heiligen / Seeligen und Gottseeligen Personen/Welche mit ihrem scheinbaren Leben/ Herrlichen Todt/ oder anderen fürtrefflichen Thaten/ Die sogenannte eigentliche Gefürstete Grafschafft Tyrol [...] gezieret haben, Augsburg 1732, Teil 2, 154-167: “Geschicht Von dem grausamen durch die Juden verübten KindsMord An dem heiligen Knäblein Andreas von Rünn", zit. n. Langer, Zwischen Vorurteil und Aggression, S. 254.

199 Anton Maximilian Heiß, Kleine Bauren-Legend Oder Heiliger Hirten und Bauren Leben, Augsburg 1734

200 Schroubek, Zur Verehrungsgeschichte des Andreas von Rinn, S. 3846.

201 Kembter, Acta pro veritate martyrii corporis et cultus publici B. Andreae Rinnesis pueruli [...]. 
Veröffentlichungen nicht der Fall gewesen. In den übrigen Kapiteln werden die Legende sowie u. a. Wunderberichte, die an Guarinonis Manuskript von 1651 angelehnt sind, wiedergegeben. Kembters Quellen-Überlegungen zur Andreas-Legende, welche in Vorbereitung zur geplanten Heiligsprechung Mitte des 18. Jahrhunderts erfolgten, waren unter anderem ein Grund, weshalb das Anderl nicht heiliggesprochen werden konnte (trotz Bemühungen der Prämonstratenser). ${ }^{202}$

1634 erlaubte Papst Urban VIII. eine öffentliche Verehrung ohne formellen Seligsprechungsakt (Beatificatio aequipollens) ${ }^{203}$ all jener "potentiell Heiligen”, die vor 1535 verstorben waren. Da dies auch für Simon von Trient ${ }^{204}$ und den "Märtyrer" aus Rinn galt, ging man zu jener Zeit wohl auch schon von einer früheren Verehrung Andreas' aus. 2051755 sprach sich Rom dann zwar auf Betreiben des Wiltener Abtes Norbert Bußjäger ${ }^{206}$ mittels der "Constitutio Beatus Andreae“207 für eine Kulterlaubnis aus (Officium proprium), ${ }^{208}$ was für die Rinner Kultstätte einen totalen Ablass bedeutete. Der Kult (Festtag ist der 12. Juli) wurde in Folge mit Messe und Brevier exklusiv für die Diözese Brixen genehmigt. ${ }^{209}$ Durch diese 1755 erfolgte offizielle Kulterlaubnis, jedoch erlebte die Anderl-Verehrung einen überregionalen Aufschwung. Dies belegen Erwähnungen in italienischen Schriften wie z. B. der von Giovanni Pietro Vitti ${ }^{210}$ von 1761. ${ }^{211}$

Ebenso erlebten in dieser Zeit religiös motivierte Volksschauspiele eine Hochblüte. Neben z. B. Passionsspielen, dem Nachempfinden des Letzten Abendmahles oder Inszenierungen zu Christi Himmelfahrt, wurde auch das Anderl von Rinn in dieser Weise popularisiert. ${ }^{212}$

Das Jesuitengymnasium Hall inszenierte bereits 1621 eine einmalige Schulaufführung in der Salzstadt, die die Geschichte des Anderl thematisierte. Das „Anderlspiel“213 erfuhr großen Zuspruch und wurde in Folge, ab Mitte des 17. Jahrhunderts bis Ende des 18. Jahrhunderts, ${ }^{214}$ als volkserzieherisches Schauspiel aufgeführt. Es war wichtig für die Verbreitung der Legende in Tirol und wurde zuerst mehrmals in Amras aufgeführt, etwas später auch in Rinn, Kitzbühel, Arzl i. Pitztal, Zirl und Imst.215 Der Spieltext der Erstaufführung von 1621 beruhte großteils auf Guarinonis Ideen und war auf Latein verfasst. Dem gegenüber war die Perioche, die für die nur mangelhaft alphabetisierten

\footnotetext{
202 Noflatscher, Jüdisches Leben in Tirol, S. 245-246.

203 Poli, Ritualmordlegenden in Tirol, S. 303.

204 Ebd., S. 302-303.

205 Holzmann, Vier Ritualmordlegenden im Vergleich, S. 70.

206 Gelmi, Geschichte der Kirche in Tirol, S. 100; Poli, Ritualmordlegenden in Tirol, S. 302-303.

207 Ebd., S. 303.

208 Ebd.

209 Ebd., S. 302-303.

210 Giovanni Pietro Vitti, Memorie storico-cronologice di varj bambini [...] martirizzati dagli Ebrei, Venezia 1761, zit. n. Schroubek, Zur Verehrungsgeschichte des Andreas von Rinn, S. 3849.

211 Ebd.

212 Gelmi, Geschichte der Kirche in Tirol, S. 195.

213 Poli, Ritualmordlegenden in Tirol, S. 302.

214 Ellen Hastaba, Vom Lied zum Spiel. Das Anderl-von-Rinn-Lied des Hippolyt Guarinoni als Vorlage für Anderl-vonRinn-Spiele, in: Johann Holzner/Oskar Putzer/Max Siller (Hrsg.), Literatur und Sprachkultur in Tirol (Innsbrucker Beiträge zur Kulturwissenschaft. Germanistische Reihe 55), Innsbruck 1997, S. 273-288, hier S. 284; Dazu: Brandstätter, Antijüdische Ritualmordvorwürfe in Trient und Tirol, S. 527.

215 Gelmi, Geschichte der Kirche in Tirol, S. 208-211.
} 
Zuschauer gedacht war, auf Deutsch abgefasst. Heinz Noflatscher geht davon aus, dass sie nicht auf Guarinoni zurückgeht, sondern auf einen Jesuitenbruder, der auch das Drama geschrieben hatte. ${ }^{216}$ In späteren Aufführungen wurde das Stück zum besseren Verständnis auf Deutsch ${ }^{217}$ aufgeführt, wobei der Text, bis auf einige wenige Adaptionen, ab 1642 auf Guarinonis Schrift "Triumph Cron [...]" basierte. ${ }^{218}$

Besonders hervorzuheben ist bei den jesuitischen Anderl-Volksschauspielen, dass neben der katholisch-religiösen-Erziehung auch die Erheiterung des Gemüts beabsichtigt war. Das Zielpublikum war nach Noflatscher vorwiegend höfischer- oder städtischer Herkunft; ${ }^{219}$ nach Langer waren es tendenziell ungebildetere Leute. ${ }^{220}$ So oder so wurden neben Katholik_innen auch „,Widersacher', ,allerley Sectische' aber auch, hohe Potanten"'221, die sich unterhalten lassen wollten, herzlich willkommen geheißen. ${ }^{222}$

Jüdinnen und Juden waren zu dieser Zeit zwar oft in der Rolle als Mörder_innen Protagonist_innen der Stücke, lebten aber zumeist nicht (mehr) in den Orten, in denen Jesuitentheater stattfanden, wobei Innsbruck eine Ausnahme darstellte. ${ }^{223}$ Die Jesuitenspiele wurden im Angesicht der sich anbahnenden Aufklärung theoretisch bereits 1752 durch Kaiserin Maria Theresia verboten und endeten praktisch 1773,224 als der Jesuitenorden durch Papst Klemens XIV. aufgehoben wurde.225 Die Reformen im religiösen Bereich, die unter Maria Theresias Sohn, Kaiser Joseph II., ihren Zenit erreichten, wurden vom Volk als tiefgreifender Einschnitt in die „Volksseele" empfunden. Deshalb wurden in vielen Fällen verbotene Riten und Kulte trotz Verbots inoffiziell weiter zelebriert. ${ }^{226}$

Natürlich brachen alle aufgezeigten Anderl-Verbreitungs-Medien, in denen auch Simon von Trient oft als zweiter "Tiroler Heiliger" mit eingebracht wurde, nicht einfach gegen Ende des 18. Jahrhunderts ab - sie veränderten sich. Waren im 17. und 18. Jahrhundert noch die Predigt von der Kanzel, Erbauungsschriften und leicht verständliche Texte mit reichlicher Bebilderung verbreitet worden, so änderte sich dies an der Wende zum 19. Jahrhundert. Ab 1800 entstanden neue Arten von Medien (z. B. Pilgerführer, Andachtsbildchen), ${ }^{227}$ die dafür sorgten, dass der Kult nicht abriss. Die Legende wurde außerdem in die in der ersten Hälfte des 19. Jahrhunderts weit verbreiteten Sagen- und Märchenbücher ${ }^{228}$ aufgenommen und bis ins 20. Jahrhundert weitergetragen. ${ }^{229}$

216 Noflatscher, Jüdisches Leben in Tirol, S. 227-228.

217 Ebd., S. 238.

218 Brandstätter, Antijüdische Ritualmordvorwürfe in Trient und Tirol, S. 526-527.

219 Ebd., S. 241.

220 Langer, „Blutbegier'ge Judenhunde streichen durch dies fromme Land....", S. 45.

221 Guarinoni, Die Grewel der Verwüstung Menschlichen Geschlechts, S. 214-215, zit. n. Hastaba, Vom Lied zum Spiel, S. 280 .

222 Ebd., S. 279-281.

223 Noflatscher, Jüdisches Leben in Tirol, S. 139-218.

224 Im 19. Jahrhundert setzten die Spiele mit großem Erfolg wieder ein. Dazu: Holzmann, Vier Ritualmordlegenden im Vergleich, S. 72.

225 Gelmi, Geschichte der Kirche in Tirol, S. 221-225.

226 Maria-Beate Eder, Absam - Ein Beispiel einer jungen Wallfahrt, phil. Diss. Innsbruck 1989, S. 30-33.

227 Langer, Zwischen Vorurteil und Aggression, S. 257.

228 Jacob Grimm/Wilhelm Grimm (Hrsg.), Deutsche Sagen. Ausgabe auf der Grundlage der ersten Auflage. Ediert und kommentiert von Heinz Rölleke (Bibliothek deutscher Klassiker 116), Frankfurt a. M. 1994, S. 376-377.

229 Schroubek, Zur Verehrungsgeschichte des Andreas von Rinn, S. 3849-3850. 


\section{Fazit}

Zusammenfassend kann festgehalten werden, dass Hippolyt Guarinoni als Schlüsselfigur für den Anderl-Kult, so wie wir ihn durch Quellen nachvollziehen können, zu bezeichnen ist. Die Gründe dafür sind maßgeblich in seiner Vita und seiner barockkatholizistisch-antijüdischen Einstellung, die auch sein Umfeld teilte, zu suchen. Aus diesen Beweggründen heraus wollte er einen Tiroler Märtyrerkult kreieren, der nach dem Muster der Simon von Trient-Legende konzipiert sein sollte. Hinweise dafür sind etwa der Tathintergrund beider Legenden und die Namenwahl der Eltern Anderls. Nichtsdestotrotz wurde veranschaulicht, dass der Kult, der zwar mit Guarinoni seinen Ausgang nahm, nicht nur an den Haller Stadtarzt geknüpft war. Dafür spricht einerseits die Verehrung eines vermutlich getöteten Rinner Knaben, die bereits vor den Ausführungen Guarinonis zum Anderl existierte. Andererseits erlebte der Andreas-Kult nach dem Ableben Hippolyt Guarinonis 1654 seine Blüte im 17. und 18. Jahrhundert. Dies wurde unter besonderer Berücksichtigung der Judensteiner Wallfahrtskirche, die heute noch von Wichtigkeit ist und in der der Anderl-Kult kritisch aufgearbeitet wurde, dargestellt. Zur weiteren Verdeutlichung wurden das dortige Wallfahrtswesen, Predigten, Prozessionen, Andachten, Gebete, Publikationen/Lieder und Volksschauspiele zum Anderl herangezogen. All dies lässt letzten Endes erkennen, dass Hippolyt Guarinoni zwar eine zentrale Rolle in Bezug auf den Andreas-Kult einnahm, dieser jedoch keinesfalls nur auf ihn beschränkt war.

\section{Literatur und Quellen}

Anselm, Sigrun, Angst und Angstprojektion in der Phantasie vom jüdischen Ritualmord, in: Rainer Erb (Hrsg.), Die Legende vom Ritualmord. Zur Geschichte der Blutbeschuldigung gegen Juden (Dokumente, Texte, Materialien 6), S. 253-265.

Brandstätter, Klaus, Antijüdische Ritualmordvorwürfe in Trient und Tirol. Neuere Forschungen zu Simon von Trient und Andreas von Rinn, in: Historisches Jahrbuch 125 (2005), S. 495-536.

Bücking, Jürgen, Kultur und Gesellschaft in Tirol um 1600. Des H. Guarinonius', Grewel der Verwüstung Menschlichen Geschlechts' (1610) als kulturgeschichtliche Quelle des frühen 17. Jahrhunderts (Historische Studien 401), Lübeck-Hamburg 1968.

Dahl, David L., Ritualmordvorwurf in Trient (1475), in: Wolfgang Benz (Hrsg.), Ereignisse, Dekrete, Kontroversen (Handbuch des Antisemitismus. Judenfeindschaft in Geschichte und Gegenwart 4), Berlin-Boston 2011, S. 356-358.

Diözese Innsbruck (Hrsg.), Judenstein. Das Ende einer Legende. Dokumentation, Innsbruck 1995.

Eder, Maria-Beate, Absam - Ein Beispiel einer jungen Wallfahrt, phil. Diss. Innsbruck 1989.

Erb, Rainer, Drittes Bild: Der „Ritualmord“, in: Julius H. Schoeps/Joachim Schlör (Hrsg.), Antisemitismus. Vorurteile und Mythen, München 1995, S. 74-79. 
Eybl, Franz M., Das Anderl von Rinn in barocken Predigten, in: Alfred Ebenbauer/Klaus Zatloukal (Hrsg.), Die Juden in ihrer mittelalterlichen Umwelt, Köln-Wien 1991, S. 27-34.

Fresacher, Bernhard, Anderl von Rinn. Ritualmord und Neuorientierung in Judenstein 1945-1995 (Mit einem Nachwort von Altbischof Reinhold Stecher), Innsbruck 1998.

Fresacher, Bernhard, Gedächtnis im Wandel. Eine interdisziplinäre systematisch-theologische Studie über die Verarbeitung von Traditionsbrüchen in der Kirche. Mit dem Fallbeispiel „Judenstein", theol. Diss. Salzburg 1995.

Gelmi, Josef, Geschichte der Kirche in Tirol. Nord-, Ost- und Südtirol, Innsbruck 2001.

Grass, Franz, Dr. Hippolytus Guarinonius zu Hoffberg und Volderthurn 1571-1654, in: Raimund Klebelsberg (Hrsg.), Hippolytus Guarinonius (1571-1654). Zur 300. Wiederkehr seines Todestages (Schlern-Schriften 126), Innsbruck 1954.

Grimm, Jacob/Grimm, Wilhelm (Hrsg.), Deutsche Sagen. Ausgabe auf der Grundlage der ersten Auflage. Ediert und kommentiert von Heinz Rölleke (Bibliothek deutscher Klassiker 116), Frankfurt a. M. 1994.

Guarinoni, Hippolyt, Begrü[n]dte Historj / Der Marter, deß Haillig = / Unschuldigen Khindtß / Andree Von Rinn / So durch die Juden, Im 1462. Jahr / Den 12. tag Julj, dem Christe[n]thumb / Zu Hoon Vnd Spott, Ermördt [...], Innsbruck 1651, Stiftsarchiv Wilten, $38 \mathrm{~A} 1$.

Guarinoni, Hippolyt, Prälaten Cron Lebens und Der Gewaltigen Thaten deß H. Caroli Borromaei [...], Freiburg i. Br. 1618.

Guarinoni, Hippolyt, Triumph Cron Marter Vnd Grabschrifft deß Heilig-Vnschuldigen Kindts ANDREAE Von Rinn [...], Innsbruck 1642.

Hastaba, Ellen, Vom Lied zum Spiel. Das Anderl-von-Rinn-Lied des Hippolyt Guarinoni als Vorlage für Anderl-von-Rinn-Spiele, in: Johann Holzner/Oskar Putzer/Max Siller (Hrsg.), Literatur und Sprachkultur in Tirol (Innsbrucker Beiträge zur Kulturwissenschaft. Germanistische Reihe 55), Innsbruck 1997, S. 273-288.

Heiß, Anton Maximilian, Kleine Bauren-Legend Oder Heiliger Hirten und Bauren Leben, Augsburg 1734.

Holzmann, Isabella, Vier Ritualmordlegenden im Vergleich. Anderl von Rinn, Simon von Trient, Ursula Pöck von Lienz, Franz Locherer von Montiggl. Mit besonderer Berücksichtigung des Andreas von Rinn, phil. Dipl. Innsbruck 2013.

Jung, Martin H., Reformation und Konfessionelles Zeitalter (1517-1648), GöttingenOakville 2012.

Jungiger, Horst, Talmudhetze, in: Wolfgang Benz (Hrsg.), Ereignisse, Dekrete, Kontroversen, (Handbuch des Antisemitismus. Judenfeindschaft in Geschichte und Gegenwart 4), Berlin-Boston 2011, S. 406-408. 
Kapferer-Prinoth, Petra/Kapferer, Martin, Rinn: Wallfahrtskirche Mariä Heimsuchung in Judenstein, in: Reinhard Rampold (Hrsg.), Kunstführer Tirol. Die 400 bedeutendsten Kunstschätze in Nord- und Osttirol, Innsbruck 2014, S. 146-147.

Kembter, Adrian, Acta pro veritate martyrii corporis et cultus publici B. Andreae Rinnesis pueruli anno MCCCCLXII. die 12. julii a judaeis in odium fidei occisi [... ], Innsbruck 1745. Kogler, Nikolaus Christian, Votivbilder aus dem östlichen Nordtirol I, phil. Diss. Innsbruck 1962.

Kogler, Nikolaus Christian, Votivbilder aus dem östlichen Nordtirol II, phil. Diss. Innsbruck 1962.

Konrad, Verena, Guarinoni, Hippolyt (Projekt Artisti Italiani Austria der Universität Innsbruck, Leitung: ao. Univ.-Prof. Peter Fidler), März 2009, [https://www.uibk.ac.at/aia/guarinoni_hippolyt.html], eingesehen 4.1.2017.

Kriegbaum, Bernhard, Petrus Canisius - ein Lebensbild (27. April), in: Jesuiten. Mitteilungen derösterreichischen Jesuiten 70 (1997), Heft 1, S. 4-6, [http://www.jesuiten.at/der-orden/ heilige-und-selige-jesuiten/detail/?no_cache=1\&tx_sihistory_pi1\%5Buid\%5D=17], eingesehen 7.1.2017.

Langer, Michael, „Blutbegier'ge Judenhunde streichen durch dies fromme Land...." Ritualmordwahn und Tiroler Volksfrömmigkeit, in: Diözese Innsbruck (Hrsg.), Judenstein. Legende ohne Ende, Innsbruck o. J., S. 31-62.

Langer, Michael, Zwischen Vorurteil und Aggression. Zum Judenbild in der deutschsprachigen katholischen Volksbildung des 19. Jahrhunderts (Lernprozeß Christen Juden 9), Freiburg i. Br. 1994.

Leeb, Rudolf, Der Streit um den wahren Glauben - Reformation und Gegenreformation in Österreich, in: Herwig Wolfram (Hrsg.), Geschichte des Christentums in Österreich. Von der Spätantike bis zur Gegenwart (Österreichische Geschichte o.A.), Wien 2003, S. 145-279.

Melzer, Gottfried, Das selige Kind Andreas von Rinn. Ein wahrer Märtyrer der katholischen Kirche, Durach 1989.

Melzer, Gottfried, Der selige Andreas von Rinn als Helfer und Wundertäter, Durach 1991.

Melzer, Gottfried, Judenstein im Licht der Wahrheit. Fehlerhaftes, Fehlendes, Bedenkliches und Beleidigendes im Buch "Judenstein - das Ende einer Legende", Bad Hall 1996. Melzer, Gottfried (Hrsg.), Wir beten zum seligen Andreas von Rinn. Unschuldiges Kind und Märtyrer. Gebete und Novene zu Ehren des seligen Kindes nebst einem Lebensabriss, Seewen 1994.

Noflatscher, Heinz, Jüdisches Leben in Tirol im 16. und 17. Jahrhundert, in: Thomas Albrich (Hrsg.), Vom Mittelalter bis 1805 (Jüdisches Leben im historischen Tirol 1), Innsbruck-Wien 2013, S. 135-246. 
Österreichische Provinz der Gesellschaft Jesu, Geschichte des Ordens, o. A., [http:// www.jesuiten.at/der-orden/geschichte-des-ordens/], eingesehen 7.1.2017.

Öttl, Herta/Franckenstein, Josef u. a., Rinn: Ehem. Wallfahrtskirche, in: Institut für österreichische Kunstforschung des Bundesdenkmalamtes (Hrsg.), Dehio Tirol (DehioHandbuch. Die Kunstdenkmäler Österreichs. Topographisches Denkmälerinventar. Tirol), Wien 1980, S. 655-657.

Palme, Rudolf, Zur spätmittelalterlichen und frühneuzeitlichen Sozial- und Rechtsgeschichte der Juden in Tirol, in: Alfred Ebenbauer/Klaus Zatloukal (Hrsg.), Die Juden in ihrer mittelalterlichen Umwelt, Köln-Wien 1991, S. 183-203.

Paulin, Karl, Die schönsten Tiroler Sagen, Innsbruck 1955.

Petzoldt, Leander, Religion zwischen Sentiment und Protest. Zur Sistierung des Kultes um „Andreas von Rinn“ in Tirol, in: Zeitschrift für Volkskunde 83 (1987), II. Halbjahresband, S. 169-192.

Poli, Roland T., Ritualmordlegenden in Tirol. Mit besonderer Berücksichtigung des Falles Franz Thomas Locherr (1744), in: Robert Rebitsch/Elena Taddei (Hrsg.), Politik - Konflikt - Gewalt (Innsbrucker Historische Studien 25), Innsbruck 2007, S. 295-319.

Schomers, Florian N., Wallfahrtskirche Mariae Heimsuchung, Judenstein, O. O. 2009.

Schreiber, Wolftraud, Simon von Trient \& Anderl von Rinn. Zwei antijüdische Lügenmärchen, in: Paul Preims/Hans Wielander (Hrsg.), Trentino. Ein Lesebuch, O. O. 2013, S. 31-35.

Schroubek, Georg R., Andreas von Rinn. Der Kult eines "heiligen Mordopfers“ im historischen Wandel, in: Österreichische Zeitschrift für Volkskunde 98 (1995), Heft 4, S. 371-396.

Schroubek, Georg R., Zur Frage der Historizität des Andreas von Rinn, in: Susanna Buttaroni/Stanislaw Musial (Hrsg.), Ritualmord. Legenden in der europäischen Geschichte, Wien-Köln-Weimar 2003, S. 173-196.

Schroubek, Georg R., Zur Tradierung und Diffusion einer europäischen Aberglaubensvorstellung, in: Rainer Erb (Hrsg.), Die Legende vom Ritualmord. Zur Geschichte der Blutbeschuldigungen gegen Juden (Dokumente, Texte, Materialien 6), Berlin 1993, S. 17-24.

Schroubek, Georg R., Zur Verehrungsgeschichte des Andreas von Rinn. Zweiter Teil, in: Das Fenster 20 (1986), Heft 39, S. 3845-3855.

Strack, Hermann L., Das Blut im Glauben und Aberglauben der Menschheit. Mit besonderer Berücksichtigung der "Volksmedizin“ und des "jüdischen Blutritus", München 1900.

Tabernig, Birgit, Die Irrationalität der Ritualmordlegenden. Entwicklungen und Hintergründe jüdischer Ritualmordlegenden des Spätmittelalters und der frühen Neuzeit mit besonderer Berücksichtigung der Lienzer Beschuldigung von 1442/43, phil. Dipl. Innsbruck 2000. 
Zach, Ignatius, Ausführliche Beschreibung der Marter Eines heiligen und unschuldigen Kinds Andreae von Rinn in Tyrol und Bistumb Brixen [...], Augsburg 1724.

Judith Dengler ist Studentin der Geschichte (MA) und Kunstgeschichte (BA) an der Universität Innsbruck. Judith.Dengler@uibk.ac.at

\section{Zitation dieses Beitrages}

Judith Dengler, Die tirolische Legende vom „Anderl von Rinn“ - Andreaskult und Wallfahrtskirche, in: historia.scribere 10 (2018), S. 211-240, [http://historia.scribere.at], eingesehen 19.6.2018 (=aktuelles Datum).

(C) Creative Commons Licences 3.0 Österreich unter Wahrung der Urheberrechte der Autorlnnen. 